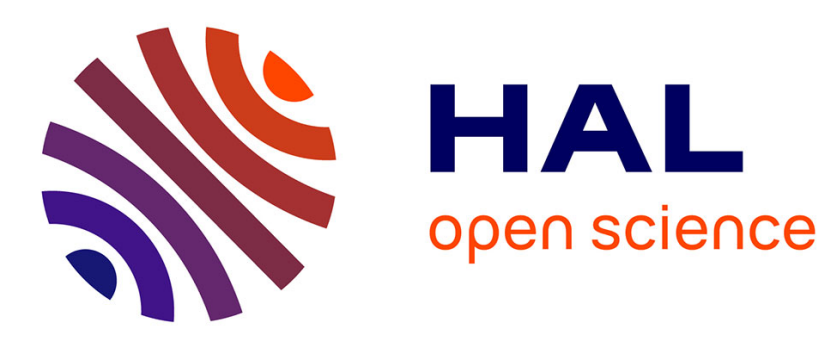

\title{
An all-speed relaxation scheme for gases and compressible materials
}

\author{
Emanuela Abbate, Angelo Iollo, Gabriella Puppo
}

\section{To cite this version:}

Emanuela Abbate, Angelo Iollo, Gabriella Puppo. An all-speed relaxation scheme for gases and compressible materials. Journal of Computational Physics, 2017, 351, pp.1-24. 10.1016/j.jcp.2017.08.052 . hal-01586863

\section{HAL Id: hal-01586863 https://hal.inria.fr/hal-01586863}

Submitted on 13 Sep 2017

HAL is a multi-disciplinary open access archive for the deposit and dissemination of scientific research documents, whether they are published or not. The documents may come from teaching and research institutions in France or abroad, or from public or private research centers.
L'archive ouverte pluridisciplinaire HAL, est destinée au dépôt et à la diffusion de documents scientifiques de niveau recherche, publiés ou non, émanant des établissements d'enseignement et de recherche français ou étrangers, des laboratoires publics ou privés. 


\title{
An all-speed relaxation scheme for gases and compressible materials
}

\author{
Emanuela Abbate ${ }^{\mathrm{a}, \mathrm{b}, \mathrm{c}}$, Angelo Iollo ${ }^{\mathrm{b}, \mathrm{c}}$, Gabriella $\mathrm{Puppo}^{\mathrm{a}}$ \\ ${ }^{a}$ Università degli Studi dell'Insubria, via Valleggio, Como 22100, Italy \\ ${ }^{b}$ Université Bordeaux, IMB, UMR 5251, F-33400 Talence, France \\ ${ }^{c}$ Equipe-projet Memphis, Inria Bordeaux-Sud Ouest, F-33400 Talence, France
}

\begin{abstract}
We present a novel relaxation scheme for the simulation of compressible material flows at all speeds. An Eulerian model describing gases, fluids and elastic solids with the same system of conservation laws is adopted. The proposed numerical scheme is based on a fully implicit time discretization, easily implemented thanks to the linearity of the transport operator in the relaxation system. The spatial discretization is obtained by a combination of upwind and centered schemes in order to recover the correct numerical viscosity in all different Mach regimes. The scheme is validated by simulating gas and liquid flows with different Mach numbers. The simulation of the deformation of compressible solids is also approached, assessing the ability of the scheme in approximating material waves in hyperelastic media.
\end{abstract}

Keywords: Low Mach limit, compressible flows, relaxation method, Eulerian elasticity, all-speed scheme

\section{Introduction}

Different physical phenomena are affected by drastic changes of the sound speed or, in general, of specific waves speeds. The occurrence of these changes may be caused for example by the geometry of the problem, e.g. a gas or a fluid flow in a nozzle. Other examples include the propagation of waves in heterogeneous compressible solids: these waves can travel at different speeds due to the local stiffness of the material.

This work focuses on the study of flows with different Mach numbers pertaining both fluid dynamics and continuum mechanics. Phenomena involving fluid flows and elastic materials deformations are investigated with 
an Eulerian approach. We introduce a monolithic model which is able to describe each material (gas, liquid or solid) with the same system of conservation equations and an appropriate general formulation of the constitutive law $[1,2,3,4,5]$. The classical Euler system used in fluid dynamics can be seen as a particular case inside this general framework.

The low Mach limit consists in observing material waves travelling with a consistently lower speed with respect to a subset of the remaining waves. In the case of fluids, it means that the flow velocity is consistently slower than the acoustic waves. In the case of an elastic solid, the deformation wave is consistently slower than the longitudinal compression waves and/or slower than the shear isochoric waves.

In general, when the Mach number tends to zero, compressible flow equations converge to incompressible equations, as it has been mathematically studied in $[6,7,8,9]$. Therefore, specific numerical problems arise when solving low Mach number flows with Godunov-type schemes [10, 11]. Firstly, the upwind discretization provides an excessive numerical viscosity on the slow waves when the Mach number tends to zero, as detailed in $[12,13]$. In these works, the importance of centering pressure gradients in the limit of small Mach numbers has then been addressed. For the present study, the excess of viscosity is observed in the stress tensor fluctuations. These fluctuations describe both the acoustic compression and the elastic deformation.

The second important issue in adopting standard explicit methods for low Mach flows is due to the enforcement of the CFL stability condition. This condition limits the time step with the space step divided by the fastest wave speed. Thus, the time step of compressible codes becomes extremely small as the incompressible regime gets closer, requiring an increasingly large computational time.

A variety of different methods have been developed to overcome these problems. A possible approach consists in choosing implicit time discretizations, which allow to avoid the acoustic CFL constraint. Several preconditioning techniques have been devised to decrease the numerical diffusion of upwind schemes at low Mach numbers in such implicit methods [14, 15, 16, 17], starting from the "artificial compressibility" technique of Chorin [18] and from Turkel's work [19, 20]. However, it is extremely difficult to handle the non-linearities of classical upwind discretizations (e.g. approximate Riemann solvers) when implicit schemes are employed.

In other works a different approach is adopted, with the focus on the pressure equations. The main idea consists in adapting classical incompressible 
schemes to compressible flows. Klein [21] proposes a semi-implicit scheme by solving explicitly the leading order contribution of the pressure and implicitly the lower orders. In $[22,23]$ different variations of all-speed schemes are proposed, based on the introduction of a suitable elliptic equation on the pressure. In order to do this, pressure gradient-type terms are introduced inside the momentum equation, allowing the splitting of the fast and the slow scales. These schemes have a formal proof of the Asymptotic Preserving (AP) property, namely their lower order expansion is a consistent and stable discretization of the incompressible limit (see also [24, 25, 26, 27]).

In the present paper, we aim at deriving a novel relaxation scheme for the simulation of compressible materials in different regimes, including the low Mach number limit. We adopt the relaxation method intoduced by Jin and Xin [28] due to its simplicity and generality. With this approach, the relaxation system is linear except for a lower order source. The linearity of the advective part is fundamental to easily implement an implicit scheme. Then, demanding acoustic constraints on the time step can be avoided.

In this framework, we introduce both centered and upwind spatial discretizations. The centered space discretization is needed in order to recover the correct limit on the stress tensor gradients when the Mach number tends to zero. The upwind discretization, on the other hand, introduces enough numerical viscosity when solving regimes with Mach number of order one. Hence, we build a convex combination of the two methods, which is based on the local Mach number of the flow.

We focus on one dimensional problems. The numerical illustration of the performances of the scheme is carried out through a comparison with standard explicit-upwind relaxation schemes as the ones derived in [28, 29]. The ability of our scheme in recovering the correct numerical viscosity is proven with several numerical tests. Our main interest is in accurately capturing the motion of the fluid or the material deformation. Thus, a lack of accuracy on the fast waves is acceptable in low Mach regimes, also because these waves carry a negligible amount of energy.

The outline of the paper is as follows. We provide a description of the Eulerian model for simulating compressible materials in Section 2. The low Mach limits that can arise are analyzed. Then, we briefly introduce and review the relaxation method in Section 3. In Section 4, the standard explicitupwind relaxation schemes are revised and then our novel implicit all-speed scheme is derived in detail. The numerical validation of the scheme is performed in Section 5, where its applicability to different regimes and materials 
is shown. Finally, conclusions are drawn in Section 6.

\section{Eulerian model for compressible materials}

The monolithic Eulerian model describing different materials with the same system of conservation laws is thoroughly discussed in several works $[1,2,3,4,5,30]$. In this section we briefly revise the principal elements of the model in 2D and then focus on the one dimensional version, studying the wave speeds and the low Mach limits.

\subsection{The 2D model}

Let $\Omega_{0} \in \mathbb{R}^{2}$ be the reference or initial configuration of a continuous medium and $\Omega_{t} \in \mathbb{R}^{2}$ the deformed configuration at time $t$. The forward characteristics $X(\xi, t)$ are defined as the image at time $t$ in the deformed configuration of a material point $\xi$ belonging to the initial configuration, i.e., $X: \Omega_{0} \times[0, T] \rightarrow \Omega_{t},(\xi, t) \mapsto X(\xi, t)$. The corresponding Eulerian velocity field $u$ is defined as $u: \Omega_{t} \times[0, T] \rightarrow \mathbb{R}^{2},(x, t) \mapsto u(x, t)$ where

$$
\left\{\begin{array}{l}
\partial_{t} X(\xi, t)=u(X(\xi, t), t) \\
X(\xi, 0)=\xi, \quad \xi \in \Omega_{0} .
\end{array}\right.
$$

The backward characteristics $Y(x, t)$ describe the continuum in the Eulerian framework: for a time $t$ and a point $x$ in the deformed configuration the corresponding initial point $\xi$ in the initial configuration is given, i.e., $Y: \Omega_{t} \times[0, T] \rightarrow \Omega_{0},(x, t) \mapsto Y(x, t)$. Relation $Y(X(\xi, t), t)=\xi$ can be differentiated with respect to time and space in order to get:

$$
\left\{\begin{array}{l}
\partial_{t} Y+u \cdot \nabla_{x} Y=0 \\
Y(x, 0)=x, \quad x \in \Omega_{t} .
\end{array}\right.
$$

Since the transformation from $X$ to $Y$ corresponds to an invertible change of variables, we have

$$
\left[\nabla_{\xi} X(\xi, t)\right]=\left[\nabla_{x} Y(x, t)\right]^{-1}
$$

At this point, one is able to compute the gradient of the deformation in the Eulerian frame via $Y$. Since the stress tensors have a direct dependence on $\left[\nabla_{x} Y\right]$, the gradient of $(1)$ is taken as a governing equation. The other 
governing equations are given by the conservation of mass, momentum and energy in the Eulerian framework (for details, see $[4,5]$ ). The conservative form of the equations of a general medium in the deformed configuration is given by

$$
\left\{\begin{array}{l}
\partial_{t} \rho+\operatorname{div}_{x}(\rho u)=0 \\
\partial_{t}(\rho u)+\operatorname{div}_{x}(\rho u \otimes u-\sigma)=0 \\
\partial_{t}\left(\left[\nabla_{x} Y\right]\right)+\nabla_{x}\left(u \cdot\left[\nabla_{x} Y\right]\right)=0 \\
\partial_{t}(\rho e)+\operatorname{div}_{x}\left(\rho e u-\sigma^{T} u\right)=0
\end{array}\right.
$$

Here $\rho$ is the density, $u$ is the Eulerian velocity field, $\left[\nabla_{x} Y\right]$ is the gradient of the backward characteristics and $\sigma$ is the Cauchy stress tensor, which is derived from the model chosen through the state law. $e$ is the total energy per unit mass and it is given by the sum of the kinetic energy and the internal energy per unit mass $\epsilon$ :

$$
e=\frac{1}{2}|u|^{2}+\epsilon
$$

The initial density $\rho(x, 0)$, the initial velocity $u(x, 0)$, the initial total energy $e(x, 0)$ and $\left[\nabla_{x} Y\right](x, 0)=I$ are given together with appropriate boundary conditions.

The internal energy can be decomposed in the following way [31]:

$$
\begin{aligned}
\epsilon & =\epsilon_{\text {vol }}(\rho(X(\xi, t), t), s(X(\xi, t), t))+\epsilon_{\text {iso }}(\operatorname{tr}(\bar{B}(\xi, t))) \\
& =\epsilon_{\text {vol }}(\rho(x, t), s(x, t))+\epsilon_{\text {iso }}(\operatorname{tr}(\bar{B}(x, t))) .
\end{aligned}
$$

$\epsilon_{v o l}$ is the purely volumetric contribution to the energy associated to the deformation (depending on the volume and on the entropy $s$ ), instead $\epsilon_{\text {iso }}$ is the purely isochoric (volume preserving) contribution. Here $B$ is the $2 \mathrm{D}$ right Cauchy-Green tensor, namely we have

$$
B=F F^{T}=\left[\nabla_{x} Y\right]^{-1}\left[\nabla_{x} Y\right]^{-T}, \quad J=\operatorname{det}\left[\nabla_{x} Y\right]^{-1}, \quad \bar{B}=\frac{B}{J} .
$$

We adopt a general constitutive law, which is able to describe gases, fluids and elastic solids at the same time. The state law reads $[4,5]$

$$
\epsilon\left(\rho, s,\left[\nabla_{x} Y\right]\right)=\underbrace{\frac{\kappa(s)}{\gamma-1}\left(\frac{1}{\rho}-b\right)^{1-\gamma}-a \rho+\frac{p_{\infty}}{\rho}}_{\text {general gas }}+\underbrace{\frac{\chi}{\rho}(\operatorname{tr} \bar{B}-2)}_{\text {neohookean solid }},
$$


where $\kappa(s)=\exp \left(s / c_{v}\right)$ (s being the entropy), $\bar{B}$ is defined in (4) and $\chi$, $p_{\infty}, \gamma, a, b$ are positive constants characterizing a given material. In the spirit of decomposition (3), the "general gas" part of the model (5) is purely volumetric. Instead, the "neohookean solid" part gives both a volumetric and an isochoric contribution.

The energy function (5) includes different physical behaviours. The first term is the general law of van der Waals gases, modeling the fact that the internal energy increases with a compression. The $p_{\infty}$ term describes the physical effect that $\epsilon$ must increase when the density is reduced. This term models the intermolecular forces that are present in liquids and solids. The last term is the classic law of a neohookean elastic material, $\chi$ being the shear elastic modulus. It accounts for finite deformations. As shown in Table 1, classical models are obtained by specific choices of the coefficients.

\begin{tabular}{llllll}
\hline Material & $\gamma$ & $a$ & $b$ & $p_{\infty}$ & $\chi$ \\
& & {$\left[\mathrm{Pa} \mathrm{m}^{6} / \mathrm{Kg}^{2}\right]$} & {$\left[\mathrm{m}^{3} / \mathrm{Kg}\right]$} & {$[\mathrm{Pa}]$} & {$[\mathrm{Pa}]$} \\
\hline Perfect gas & 1.4 & 0 & 0 & 0 & 0 \\
Van der Waals gas & 1.4 & 5 & $10^{-3}$ & 0 & 0 \\
Stiffened gas (water) & 4.4 & 0 & 0 & $6.8 \cdot 10^{8}$ & 0 \\
Elastic solid (copper) & 4.22 & 0 & 0 & $3.42 \cdot 10^{10}$ & $5 \cdot 10^{10}$ \\
\hline
\end{tabular}

Table 1: Typical parameters for different materials.

The Cauchy stress tensor $\sigma$ is then given by (see [31] for the derivation):

$$
\left\{\begin{array}{l}
\sigma\left(\rho, s,\left[\nabla_{x} Y\right]\right)=-p(\rho, s) I+2 \chi J^{-1}\left(\bar{B}-\frac{\operatorname{tr} \bar{B}}{2} I\right) \\
p(\rho, s)=-p_{\infty}-a \rho^{2}+k(s)\left(\frac{1}{\rho}-b\right)^{-\gamma} .
\end{array}\right.
$$

We remark that when a perfect gas is modeled, it suffices to impose $a=b=0$ and $\chi=p_{\infty}=0$ (as seen in Table 1). The Euler system with the perfect gas state law closure is easily recovered. For the sake of simplicity, in all the computations and test cases that follow, we will take $a=b=0$.

The computation of the characteristic speeds of system (2) closed by state law (5) is detailed in previous works as [4, 5]. By considering smooth flow, the energy equation is substituted by the conservation of entropy. Let $x=\left(x_{1}, x_{2}\right)$ be the coordinates in the canonical basis of $\mathbb{R}^{2}, u=\left(u_{1}, u_{2}\right)$ the velocity components. $Y_{, j}^{i}$ are the components of the tensor $\left[\nabla_{x} Y\right]$ (the 
superscript ${ }^{i}$ indicates the component of $Y$ and the subscript ${ }_{j}$ indicates the direction along which the derivative of $Y$ is calculated) and $\sigma^{i j}$ the components of the stress tensor $\sigma$. By considering variations only along the $x_{1}$ direction for simplicity, the eigenvalues get the following general expression $[4]$ :

$$
\Lambda=\left\{u_{1}, u_{1}, u_{1} \pm \sqrt{\frac{\mathcal{A}_{1} \pm \sqrt{\mathcal{A}_{2}}}{\rho}}\right\}
$$

with

$$
\left\{\begin{array}{l}
\mathcal{A}_{1}=\frac{\rho c^{2}}{2}+\chi(\alpha+\beta) \\
\mathcal{A}_{2}=\left(\frac{\rho c^{2}}{2}+\chi(\alpha-\beta)\right)^{2}+4 \chi^{2} \delta^{2},
\end{array}\right.
$$

where $c$ is the sound speed and where we have introduced the notation

$$
\left\{\begin{array}{l}
\alpha=\left(Y_{, 1}^{1}\right)^{2}+\left(Y_{, 1}^{2}\right)^{2} \\
\beta=\left(Y_{, 2}^{1}\right)^{2}+\left(Y_{, 2}^{2}\right)^{2} \\
\delta=Y_{, 1}^{1} Y_{, 2}^{1}+Y_{, 1}^{2} Y_{, 2}^{2}
\end{array}\right.
$$

\subsection{One dimensional problem formulation}

In the present study, we focus on the variations only along direction $x_{1}$, thus considering the problem in one dimension. Deformations are still considered in both the two directions, but the derivatives along $x_{2}$ are equal to zero. Tensor $\left[\nabla_{x} Y\right]$ reduces to

$$
\left[\nabla_{x} Y\right]=\left[\begin{array}{cc}
Y_{, 1}^{1} & 0 \\
Y_{, 1}^{2} & 1
\end{array}\right]
$$

The problem can be further simplified, since $\rho(x, t)=\operatorname{det}\left(\left[\nabla_{x} Y\right](x, t)\right) \rho_{0}(x)$, where $\rho_{0}$ is the initial density. Thanks to this, the equation on $Y_{, 1}^{1}$ is equivalent to conservation of mass and thus redundant, having $Y_{, 1}^{1}=\rho / \rho_{0}$.

In this framework, the Cauchy stress tensor (6) has the following two nonzero components, which are the normal and the tangential stress respectively:

$$
\begin{aligned}
& \sigma^{11}=-p(\rho, s)+2 \chi J^{-1}\left(\bar{B}^{11}-\frac{\operatorname{tr} \bar{B}}{2}\right)=-p(\rho, s)+\chi\left(1-\left(Y_{, 1}^{2}\right)^{2}-\left(\rho / \rho_{0}\right)^{2}\right) \\
& \sigma^{21}=2 \chi J^{-1} \bar{B}^{21}=-2 \chi Y_{, 1}^{2} .
\end{aligned}
$$


We have then the following compact form for the 1D Eulerian model:

$$
\partial_{t} \boldsymbol{\psi}+\partial_{x_{1}} \mathbf{F}(\boldsymbol{\psi})=0,
$$

where the vector of conservative variables and the vector of fluxes are both in $\mathbb{R}^{5}$ and read

$$
\boldsymbol{\psi}=\left[\begin{array}{c}
\rho \\
\rho u_{1} \\
\rho u_{2} \\
Y_{, 1}^{2} \\
\rho e
\end{array}\right], \quad \mathbf{F}(\boldsymbol{\psi})=\left[\begin{array}{c}
\rho u_{1} \\
\rho u_{1}^{2}-\sigma^{11} \\
\rho u_{1} u_{2}-\sigma^{21} \\
u_{1} Y_{1}^{2}+u_{2} \\
\left(\rho e-\sigma^{11}\right) u_{1}-\sigma^{21} u_{2}
\end{array}\right] .
$$

\subsubsection{Non-dimensionalization}

In order to non-dimensionalize system (9), we introduce the two speeds:

- the speed of sound, which can be computed in the following way:

$$
c\left(\rho, s, \nabla_{x} Y\right)=\sqrt{\left.\frac{\partial p}{\partial \rho}\right|_{s=c o n s t}}=\sqrt{\gamma k(s) \rho^{\gamma-1}}=\sqrt{\frac{\gamma}{\rho}\left(p+p_{\infty}\right)} .
$$

- an "isochoric elastic speed", which can be defined as:

$$
u_{i s o}=\sqrt{\frac{2 \chi}{\rho}} .
$$

By using these two speeds, two different scales can be distinguished, thus two different "Mach numbers" are defined:

- the classical acoustic Mach number, which is the ratio between the acoustic speed and the advective velocity, defined as

$$
M=\frac{u_{1}}{c}
$$

- an isochoric Mach number, which is the ratio between the isochoric speed (11) and the advective velocity, defined as

$$
M_{\chi}=\frac{u_{1}}{u_{\text {iso }}}=\sqrt{\frac{\rho u_{1}^{2}}{2 \chi}} .
$$


Inside system (9), we use the sound speed $c$ to non-dimensionalize the pressure, a reference $\bar{\chi}$ to non-dimensionalize the shear modulus and a reference normal velocity $\overline{u_{1}}$ to non-dimensionalize both $u_{1}$ and $u_{2}$. Having introduced the two Mach numbers $M$ and $M_{\chi}$, the non-dimensional system reads:

$$
\left\{\begin{array}{l}
\partial_{t} \rho+\partial_{x}\left(\rho u_{1}\right)=0 \\
\partial_{t}\left(\rho u_{1}\right)+\partial_{x}\left(\rho u_{1}^{2}\right)+\frac{\partial_{x} p}{M^{2}}-\frac{\chi}{2} \frac{\partial_{x}\left(1-\left(Y_{, 1}^{2}\right)^{2}-\left(\rho / \rho_{0}\right)^{2}\right)}{M_{\chi}^{2}}=0 \\
\partial_{t}\left(\rho u_{2}\right)+\partial_{x}\left(\rho u_{1} u_{2}\right)+\chi \frac{\partial_{x} Y_{, 1}^{2}}{M_{\chi}^{2}}=0 \\
\partial_{t}\left(Y_{, 1}^{2}\right)+\partial_{x}\left(u_{1} Y_{, 1}^{2}+u_{2}\right)=0 \\
\partial_{t}\left(\frac{1}{2} \rho u^{2}+\frac{p+\gamma p_{\infty}}{M^{2}(\gamma-1)}+\frac{\chi(\operatorname{tr} \bar{B}-2)}{2 M_{\chi}^{2}}\right)+\partial_{x}\left(\frac{1}{2} \rho|u|^{3}+\frac{\gamma\left(p+\gamma p_{\infty}\right)}{M^{2}(\gamma-1)} u_{1}\right)+ \\
\frac{\chi}{2 M_{\chi}^{2}} \partial_{x}\left[\left(\operatorname{tr} \bar{B}-2-\chi\left(1-\left(Y_{, 1}^{2}\right)^{2}-\left(\rho / \rho_{0}\right)^{2}\right)\right) u_{1}+2 \chi Y_{, 1}^{2} u_{2}\right]=0 .
\end{array}\right.
$$

Here all variables are non-dimensional (in order to simplify the notation we did not introduce "ad hoc" symbols for non-dimensional variables).

\subsubsection{Low Mach limits and wave speeds}

For gas dynamics problems, the low Mach limit stiffness is brought by the pressure gradients, which are of the order $\mathcal{O}\left(1 / M^{2}\right)$. In our Eulerian model, non-dimensionalization (14) shows that if at least one between $M$ and $M_{\chi}$ tends to zero the problem becomes stiff due to the gradients of the Cauchy stress tensor $\sigma$. The gradient of the part of $\sigma$ depending on the pressure is of the order $\mathcal{O}\left(1 / M^{2}\right)$, exactly as in gas dynamics problems. The gradient of the elastic part of $\sigma$ (the one depending on $B$ and $\left[\nabla_{x} Y\right]$ ) is of the order $\mathcal{O}\left(1 / M_{\chi}^{2}\right)$. Therefore, when $M \rightarrow 0$ is verified, the stiffness is only due to the pressure gradients and when $M_{\chi} \simeq M \rightarrow 0$ the stiffness is due to both the pressure gradients and the elastic deformation gradients.

In the 1D formulation (9) we distinguish five waves $[4,5]$ :

1. two longitudinal waves (relative to the normal stress) with speed

$$
\lambda_{1,5}=u_{1} \pm \sqrt{c^{2} / 2+\chi / \rho(\alpha+\beta)+1 / \rho \sqrt{\left(\rho c^{2} / 2+\chi(\alpha-\beta)\right)^{2}+4 \chi^{2} \delta^{2}}} ;
$$


2. two shear waves (relative to the tangential stress) with speed

$$
\lambda_{2,4}=u_{1} \pm \sqrt{c^{2} / 2+\chi / \rho(\alpha+\beta)-1 / \rho \sqrt{\left(\rho c^{2} / 2+\chi(\alpha-\beta)\right)^{2}+4 \chi^{2} \delta^{2}}} ;
$$

3. one material wave with speed $\lambda_{3}=u_{1}$, namely the flow velocity.

In (15)-(16) we are using the notation introduced in (8). It is clear that longitudinal waves are always faster than shear waves. By setting $\chi=0$, we recover the wave speeds of the classic Euler system for gases and liquids: longitudinal waves are only acoustic and shear waves are not present.

According to notation (8), in the 1D formulation we have $\delta=Y_{, 1}^{2}$. Then, in the case of a small deformation, we can set $\delta \simeq 0$. With this "small deformation approximation", the speeds (16) of shear waves reduce to only the isochoric contribution proportional to $\chi$. Longitudinal waves still consist in a compression and an elastic contribution. Then, two different "low Mach" limits can be distinguished:

1. acoustic and shear low Mach regime: $M \ll 1$ and $M_{\chi} \ll 1$. Both the pressure gradient and the elastic deformations (through the gradients of $\left.\left[\nabla_{x} Y\right]\right)$ are responsible for the stiffness of this regime. Having $\mathcal{O}(M) \simeq$ $\mathcal{O}\left(M_{\chi}\right)$ means $c \simeq u_{\text {iso }}$ and $p+p_{\infty} \simeq \chi$. Thus, longitudinal and shear waves are all consistently faster than the material wave;

2. only acoustic low Mach regime: $M \ll 1$ and $M \ll M_{\chi}$. The pressure gradient is the only responsible for the stiffness of this regime. Having $c \gg\left|u_{1}\right|$ and $c \gg u_{\text {iso }}$ corresponds to $p+p_{\infty} \gg \chi$. Longitudinal waves are consistently faster than all the other waves.

For the considered Eulerian model, the low Mach regime consists in having a low acoustic Mach number, as for the classic Euler system for perfect gases. In this limit, the isochoric Mach number can also be low (of the order of the Mach number), thus introducing additional stiffness to the problem.

\section{The relaxation method}

The resolution of the low Mach regime is difficult due to the stiffness of the Cauchy stress tensor gradients (see sections 2.2.1-2.2.2) and due to the high velocities of acoustic and shear waves. To allow for an efficient and robust numerical procedure, we adopt a relaxation approach (for references on the relaxation method see $[32,33,28,34,35,36])$. In the same spirit of $[28,37]$, 
we build a linear hyperbolic relaxation system that approximates the original system (9) with a small dissipative correction. Due to the linearity of the advection terms, the numerical solution does not require the introduction of sophisticated solvers, allowing for a simpler implementation of implicit time discretizations. This is our main reason for choosing this specific relaxation approach.

By introducing the relaxation variables vector $\mathbf{v} \in \mathbb{R}^{5}$ (same dimension of the conservative variables vector $\boldsymbol{\psi}$ of system (9)), the relaxation system reads:

$$
\left\{\begin{array}{l}
\partial_{t} \boldsymbol{\psi}+\partial_{x} \mathbf{v}=0 \\
\partial_{t} \mathbf{v}+\mathbf{A} \partial_{x} \boldsymbol{\psi}=\frac{1}{\eta}(\mathbf{F}(\boldsymbol{\psi})-\mathbf{v}), \quad \eta>0
\end{array}\right.
$$

where $\mathbf{A}=\operatorname{diag}\left\{a_{i}\right\}, i=1, . ., 5$ is a positive diagonal matrix. The small positive parameter $\eta$ is called relaxation rate. The right hand side of the second equation is a stiff lower order source term and it is the only nonlinear part.

With a Chapman-Enksog expansion of the variables for small $\eta$, the behaviour of system (17) at different orders is assessed. At leading order (small relaxation limit), the original system is recovered, with the relaxation variables equal to the fluxes:

$$
\left\{\begin{array}{l}
\mathbf{v}=\mathbf{F}(\boldsymbol{\psi}) \\
\partial_{t} \boldsymbol{\psi}+\partial_{x} \mathbf{F}(\boldsymbol{\psi})=0
\end{array}\right.
$$

The first order approximation in the expansion is the following:

$$
\left\{\begin{array}{l}
\mathbf{v}=\eta\left(\mathbf{A}-\mathbf{F}^{\prime}(\boldsymbol{\psi})^{2}\right) \partial_{x} \boldsymbol{\psi} \\
\partial_{t} \boldsymbol{\psi}+\partial_{x} \mathbf{F}(\boldsymbol{\psi})=\eta \partial_{x}\left(\left(\mathbf{A}-\mathbf{F}^{\prime}(\boldsymbol{\psi})^{2}\right) \partial_{x} \boldsymbol{\psi}\right)
\end{array}\right.
$$

where $\mathbf{F}^{\prime}(\boldsymbol{\psi})$ is the Jacobian matrix of the flux function. In order to ensure the dissipative nature of system (19), it is necessary to respect the Liu subcharacteristic condition $[32,38]$

$$
\mathbf{A}-\mathbf{F}^{\prime}(\boldsymbol{\psi})^{2} \geq 0 \quad \forall \boldsymbol{\psi}
$$

when building the relaxation system. For $\boldsymbol{\psi}$ varying in a bounded domain, this condition is satisfied by choosing $\mathbf{A}=\operatorname{diag}\left\{a_{i}\right\}, i=1, . ., 5$ sufficiently large. The construction of the relaxation matrix $\mathbf{A}$ is detailed in Sec. 3.1. 
Initial and boundary conditions have to be imposed in a way that initial and boundary layers are not introduced [28]. Thus, these conditions need to be consistent with the equilibrium state (18).

The relaxation approach can be extended to multi-dimensional problems, as described in [28]. The method consists in a "dimension-by-dimension" relaxation of the fluxes, which allows to reduce the problem complexity.

\subsection{The relaxation matrix}

The relaxation matrix $\mathbf{A}$ is diagonal and it is built by imposing the subcharacteristic condition (20). The wave speeds of the relaxation system are the following

$$
\mu_{j}= \pm \sqrt{a_{i}}, i=1, . .5, j=1, . .10 .
$$

Condition (20) states that the eigenvalues $\lambda_{i}$ of the original system need to lie between the eigenvalues $\mu_{j}$ of the relaxation system. On the other hand, the CFL constraint has to be enforced on the speeds (21) of the relaxation system. Therefore, the smallest A satisfying (20) is needed.

In the present work, we avoid the easy choice $\mathbf{A}=a \mathbf{I}, a$ being a constant, in order to have distinct eigenvalues (21). This means reproducing all the wave speeds of the original system. In particular, we construct $\mathbf{A}$ by apriori estimating the wave speeds $\lambda_{i}$ of each specific problem. Then, we take the maximum over the domain for every speed: $\mathbf{A} \simeq \boldsymbol{\Lambda}_{\max }^{2}$, where $\boldsymbol{\Lambda}_{\max }=\operatorname{diag}\left\{\max _{x} \lambda_{i}\right\}$. The jacobian of the flux can be diagonalized as $\mathbf{F}^{\prime}(\boldsymbol{\psi})^{2}=\mathbf{R} \boldsymbol{\Lambda}^{2} \mathbf{R}^{-1}$, where $\mathbf{R}$ is the matrix of the right eigenvectors and $\boldsymbol{\Lambda}=\operatorname{diag}\left\{\lambda_{i}\right\}$. This way, the first order correction derived in Eq. (19) becomes

$$
\partial_{t} \boldsymbol{\psi}+\partial_{x} \mathbf{F}(\boldsymbol{\psi}) \simeq \eta\left(\boldsymbol{\Lambda}_{\max }^{2}-\mathbf{R} \boldsymbol{\Lambda}^{2} \mathbf{R}^{-1}\right) \partial_{x x} \boldsymbol{\psi} .
$$

The matrix $\boldsymbol{\Lambda}_{\max }^{2}-\mathbf{R} \boldsymbol{\Lambda}^{2} \mathbf{R}^{-1}$ is positive definite, thus the subcharacteristic condition is respected. Moreover, we can control and limit the diffusion of the relaxation by approximating all the original waves.

The a-priori estimation of the eigenvalues can be easily performed, since the sound speed and the elastic coefficients of the most common materials are well-known. The explicit formula for the eigenvalues is given in Sec. 2 .

\section{Numerical schemes}

System (17) is discretized with finite volumes on a Cartesian mesh. For one dimensional problems, let $\Delta x=x_{i+1 / 2}-x_{i-1 / 2}$ be the grid spacing and 
$\Delta t=t_{n+1}-t_{n}$ the time stepping. $\mathbf{w}_{i}^{n}$ denotes the approximate cell average of a quantity $\mathbf{w}$ in the cell $\left[x_{i-1 / 2}, x_{i+1 / 2}\right]$ at time $t_{n}$ and $\mathbf{w}_{i+1 / 2}^{n}$ denotes the approximate point value of $\mathbf{w}$ in $x=x_{i+1 / 2}$ and at $t=t_{n}$.

For the discussion of the numerical schemes it is convenient to treat the spatial discretization and the time discretization separately, as in the method of lines. With this approach, we briefly revisit the standard explicit relaxation scheme of [28]. Then, we present our novel implicit relaxation scheme, designed with the aim of solving problems with Mach numbers ranging from very small to order of unity. We point out that in deriving the numerical scheme only the acoustic Mach number $M$ is used, because it is always lower than $M_{\chi}$.

\subsection{Standard explicit relaxation scheme}

Jin and Xin propose a scheme where the space derivatives are explicit and only the stiff source relaxation term is implicit [28]. This discretization falls in the IMEX schemes class, which are specifically designed for problems where a stiff part is present and are widely used for relaxation systems (see, for example, [29, 39, 40, 41]).

At first order, the relaxation system is discretized in time as follows:

$$
\left\{\begin{array}{l}
\frac{\boldsymbol{\psi}^{n+1}-\boldsymbol{\psi}^{n}}{\Delta t}+\partial_{x} \mathbf{v}^{n}=0 \\
\frac{\mathbf{v}^{n+1}-\mathbf{v}^{n}}{\Delta t}+\mathbf{A} \partial_{x} \boldsymbol{\psi}^{n}=\frac{1}{\eta}\left(\mathbf{F}\left(\boldsymbol{\psi}^{n+1}\right)-\mathbf{v}^{n+1}\right)
\end{array}\right.
$$

The solution can be approached in a sequential manner: the unknowns $\boldsymbol{\psi}^{n+1}$ are calculated by solving the first equation and then are put inside the stiff source term to compute $\mathbf{v}^{n+1}$. This means that at every time step the relaxation variables $\mathbf{v}^{n+1}$ are projected on the fluxes $\mathbf{F}\left(\boldsymbol{\psi}^{n+1}\right)$. At second order, we adopt the IMEX scheme proposed in [29] (Butcher tableau of Table 2).

$$
\begin{aligned}
& \begin{array}{ll}
\text { (a) Explicit } & \text { (b) Implicit }
\end{array} \\
& \begin{array}{cc}
0 & 0 \\
1 & 0 \\
\hline 1 / 2 & 1 / 2
\end{array} \quad \begin{array}{ccc}
\gamma & 0 \\
1-2 \gamma & \gamma \\
\hline 1 / 2 & 1 / 2
\end{array}
\end{aligned}
$$

Table 2: Butcher tableau of the IMEX scheme proposed in [29], $\gamma=1-1 / \sqrt{2}$. 


\subsubsection{Upwind spatial discretization}

In general, the spatial discretization for system (17) reads

$$
\left\{\begin{array}{l}
\partial_{t} \boldsymbol{\psi}_{i}+\frac{\mathbf{v}_{i+1 / 2}-\mathbf{v}_{i-1 / 2}}{\Delta x}=0 \\
\partial_{t} \mathbf{v}_{i}+\mathbf{A} \frac{\boldsymbol{\psi}_{i+1 / 2}-\boldsymbol{\psi}_{i-1 / 2}}{\Delta x}=\frac{1}{\eta}\left(\mathbf{F}\left(\boldsymbol{\psi}_{i}\right)-\mathbf{v}_{i}\right)
\end{array}\right.
$$

For sufficiently accurate space discretizations, this approximation has an accuracy of $\mathcal{O}\left(\Delta x^{2}\right)$, because the flux is averaged in the following way:

$\mathbf{F}\left(\boldsymbol{\psi}_{i}\right)=\mathbf{F}\left(\frac{1}{\Delta x} \int_{x_{i-1 / 2}}^{x_{i+1 / 2}} \boldsymbol{\psi} d x\right)=\frac{1}{\Delta x} \int_{x_{i-1 / 2}}^{x_{i+1 / 2}} \mathbf{F}(\boldsymbol{\psi}) d x+\mathcal{O}\left(\Delta x^{2}\right)=\mathbf{F}_{i}+\mathcal{O}\left(\Delta x^{2}\right)$

The variables at the interfaces $x_{i+1 / 2}$ inside (23) have to be computed with an "ad hoc" stable scheme. For an explicit time stepping, a possible choice leading to a stable scheme is the upwind discretization.

We build the upwind scheme as in [28]. System (17) has two characteristic variables $\mathbf{v} \pm \mathbf{A}^{1 / 2} \boldsymbol{\psi}$, travelling at the frozen speeds $\pm \mathbf{A}^{1 / 2}$. The upwind spatial approximation is applied to the linear system on the two characteristic variables, obtaining the interface values as follows:

$$
\left\{\begin{array}{l}
\boldsymbol{\psi}_{i+1 / 2}=\frac{1}{2}\left(\boldsymbol{\psi}_{i+1}+\boldsymbol{\psi}_{i}\right)-\frac{1}{2} \mathbf{A}^{-1 / 2}\left(\mathbf{v}_{i+1}-\mathbf{v}_{i}\right) \\
\mathbf{v}_{i+1 / 2}=\frac{1}{2}\left(\mathbf{v}_{i+1}+\mathbf{v}_{i}\right)-\frac{1}{2} \mathbf{A}^{1 / 2}\left(\boldsymbol{\psi}_{i+1}-\boldsymbol{\psi}_{i}\right)
\end{array}\right.
$$

Plugging this into discretization (23) gives the first order upwind approximation:

$$
\left\{\begin{array}{l}
\frac{\mathbf{v}_{i+1 / 2}-\mathbf{v}_{i-1 / 2}}{\Delta x}=\frac{1}{2 \Delta x}\left(\mathbf{v}_{i+1}-\mathbf{v}_{i-1}\right)-\frac{\mathbf{A}^{1 / 2}}{2 \Delta x}\left(\boldsymbol{\psi}_{i+1}-2 \boldsymbol{\psi}_{i}+\boldsymbol{\psi}_{i-1}\right) \\
\frac{\boldsymbol{\psi}_{i+1 / 2}-\boldsymbol{\psi}_{i-1 / 2}}{\Delta x}=\frac{1}{2 \Delta x}\left(\boldsymbol{\psi}_{i+1}-\boldsymbol{\psi}_{i-1}\right)-\frac{\mathbf{A}^{-1 / 2}}{2 \Delta x}\left(\mathbf{v}_{i+1}-2 \mathbf{v}_{i}+\mathbf{v}_{i-1}\right)
\end{array}\right.
$$

For a second order approximation, a Van Leer MUSCL (Monotonic UpstreamCentered Scheme for Conservation Laws) scheme is employed.

The presented upwind discretization is classically used in explicit relaxation schemes. The explicit-upwind relaxation scheme is stable provided that the stability CFL condition on the fastest wave (acoustic CFL) is verified [28]. 


\subsection{Implicit relaxation scheme}

We propose a fully implicit relaxation scheme, with the goal of getting rid of acoustic CFL constraints. The linearity of the spatial derivatives in the relaxation system allows for a straightforward use of implicit time discretizations.

The implicit time discretization at first order is a simple backward Euler scheme and reads:

$$
\left\{\begin{array}{l}
\frac{\boldsymbol{\psi}^{n+1}-\boldsymbol{\psi}^{n}}{\Delta t}+\partial_{x} \mathbf{v}^{n+1}=0 \\
\frac{\mathbf{v}^{n+1}-\mathbf{v}^{n}}{\Delta t}+\mathbf{A} \partial_{x} \boldsymbol{\psi}^{n+1}=\frac{1}{\eta}\left(\mathbf{F}\left(\boldsymbol{\psi}^{n+1}\right)-\mathbf{v}^{n+1}\right)
\end{array}\right.
$$

For a second order implicit approximation, a BDF (Backward Differentiation Formula) of second order is adopted.

The treatment of the non-linear fluxes $\mathbf{F}(\boldsymbol{\psi})$ is dealt with applying one iteration of the Newton's method, namely the fluxes are approximated with a Taylor expansion in the following way:

$$
\mathbf{F}\left(\boldsymbol{\psi}^{n+1}\right)=\mathbf{F}\left(\boldsymbol{\psi}^{n}\right)+\mathbf{F}^{\prime}\left(\boldsymbol{\psi}^{n}\right)\left(\boldsymbol{\psi}^{n+1}-\boldsymbol{\psi}^{n}\right) .
$$

$\mathbf{F}^{\prime}\left(\boldsymbol{\psi}^{n}\right)$ is the Jacobian of the flux and can be computed analytically. For system (9) it reads

$\mathbf{F}^{\prime}(\psi)=\left[\begin{array}{ccccc}0 & 1 & 0 & 0 & 0 \\ -u_{1}^{2}-\sigma_{, \psi_{1}}^{11} & 2 u_{1}-\sigma_{, \psi_{2}}^{11} & -\sigma_{, \psi_{3}}^{11} & -\sigma_{, \psi_{4}}^{11} & -\sigma_{, \psi_{5}}^{11} \\ -u_{1} u_{2} & u_{2} & u_{1} & 2 \chi & 0 \\ \frac{u_{1} Y_{, 1}^{2}+u_{2}}{\rho} & \frac{Y_{, 1}^{2}}{\rho} & \frac{1}{\rho} & u_{1} & 0 \\ \frac{-E u_{1}+\sigma^{11} u_{1}}{\rho}-u_{1} \sigma_{, \psi_{1}}^{11}+\frac{\sigma^{21} u_{2}}{\rho} & \frac{E-\sigma^{11}}{\rho}-u_{1} \sigma_{, \psi_{2}}^{11} & -u_{1} \sigma_{, \psi_{3}}^{11}-\frac{\sigma^{21}}{\rho} & -\sigma_{, \psi_{4}}^{11} u_{1}+2 \chi u_{2} & u_{1}\left(1-\sigma_{, \psi_{5}}^{11}\right)\end{array}\right]$,

where $\sigma_{\psi_{i}}^{j k}$ stands for the derivative of the ${ }^{j k}, j, k=1,2$ component of the tensor $\sigma$ with respect to the conservative variable $\psi_{i}, i=1, . .5$. In deriving (27), we have used the fact that $\sigma_{, \psi_{1}}^{21}=\sigma_{, \psi_{2}}^{21}=\sigma_{, \psi_{3}}^{21}=\sigma_{, \psi_{5}}^{21}=0$ and $\sigma_{, \psi_{4}}^{21}=$ $-2 \chi$. The derivatives of $\sigma^{11}$ have the following expressions:

$$
\begin{array}{rlrl}
\sigma_{, \psi_{1}}^{11} & =-(\gamma-1)\left(\frac{1}{2}\left(u_{1}^{2}+u_{2}^{2}\right)-\frac{2 \chi \rho}{\rho_{0}^{2}}+\frac{2 \chi}{\rho_{0}}\right)-\frac{2 \chi \rho}{\rho_{0}^{2}}, \\
\sigma_{, \psi_{2}}^{11} & =(\gamma-1) u_{1}, & \sigma_{, \psi_{3}}^{11} & =(\gamma-1) u_{2}, \\
\sigma_{, \psi_{4}}^{11} & =(\gamma-1) 2 \chi Y_{, 1}^{2}-2 \chi Y_{, 1}^{2}, & \sigma_{, \psi_{5}}^{11} & =-(\gamma-1) .
\end{array}
$$




\subsubsection{Centered spatial discretization}

It has been proved that upwind spatial discretizations present an excessive numerical viscosity when approximating low Mach phenomena $[12,13]$. In the case of the Euler equations, these discretizations lead to pressure fluctuations of order $\mathcal{O}(M)$, while in the continuous case the pressure fluctuations are of order $\mathcal{O}\left(M^{2}\right)$ (see system (14)). Instead, centered spatial discretizations of the pressure gradient in the Euler equations avoid the excess of viscosity on any mesh when dealing with low Mach regimes [13, 42]. In the adopted Eulerian model, this corresponds to centering the stiff parts of $\sigma$ in the non-dimensional system (14).

A centered discretization of the spatial derivatives can be employed without stability problems in the implicit scheme just introduced. The interface values inside (23) are computed as follows:

$$
\left\{\begin{array}{l}
\boldsymbol{\psi}_{i+1 / 2}=\frac{1}{2}\left(\boldsymbol{\psi}_{i+1}+\boldsymbol{\psi}_{i}\right) \\
\mathbf{v}_{i+1 / 2}=\frac{1}{2}\left(\mathbf{v}_{i+1}+\mathbf{v}_{i}\right)
\end{array}\right.
$$

Then, the centered implicit scheme reads

$$
\left\{\begin{array}{l}
\frac{\boldsymbol{\psi}_{i}^{n+1}-\boldsymbol{\psi}_{i}^{n}}{\Delta t}+\frac{\mathbf{v}_{i+1}^{n+1}-\mathbf{v}_{i-1}^{n+1}}{2 \Delta x}=0 \\
\frac{\mathbf{v}_{i}^{n+1}-\mathbf{v}_{i}^{n}}{\Delta t}+\mathbf{A} \frac{\boldsymbol{\psi}_{i+1}^{n+1}-\boldsymbol{\psi}_{i-1}^{n+1}}{2 \Delta x}=\frac{1}{\eta}\left(\mathbf{F}\left(\boldsymbol{\psi}_{i}^{n+1}\right)-\mathbf{v}_{i}^{n+1}\right) .
\end{array}\right.
$$

This scheme possesses the following zero relaxation limit $\eta \rightarrow 0^{+}$:

$$
\frac{\boldsymbol{\psi}_{i}^{n+1}-\boldsymbol{\psi}_{i}^{n}}{\Delta t}+\frac{\mathbf{F}\left(\boldsymbol{\psi}_{i+1}^{n+1}\right)-\mathbf{F}\left(\boldsymbol{\psi}_{i-1}^{n+1}\right)}{2 \Delta x}=0
$$

which is a consistent and unconditionally stable discretization of system (9). Unconditional stability is easily proven for linear advection with the Von Neumann stability analysis [43]. Therefore, scheme (28) is unconditionally stable, namely a stability condition on the time step is not required. Moreover, by centering the whole fluxes, also the gradient of $\sigma$ is centered. Hence, the accuracy in space of scheme (29) does not depend on the Mach number.

\subsubsection{Hybrid spatial discretization}

The centered approximation (28) accurately solves low Mach flows, because the correct Mach number order on the stress tensor gradients is respected by the scheme. Nevertheless, we aim at deriving an all-speed scheme. 
When solving problems at high velocities (Mach number of order one or more), the centered discretization does not provide enough numerical viscosity, thus spurious numerical oscillations can arise. In this spirit, an hybrid spatial discretization is introduced, through a convex combination of the upwind and centered schemes described in Secs. 4.1.1-4.2.1. The combination is based on the local Mach number of the specific flow.

Let $\mathbf{u}$ be a general variable. For the sake of simplicity, we introduce the notation $\mathbf{D}\left(\partial_{x} \mathbf{u}\right) \simeq \partial_{x} \mathbf{u}, \mathbf{D}\left(\partial_{x} \mathbf{u}\right)$ being the numerical discretization of the spatial derivative of $\mathbf{u}$. The main idea of the scheme consists in defining the hybrid discretization $\mathbf{D}\left(\partial_{x} \mathbf{u}\right)_{h y b}$ as follows:

$$
\mathbf{D}\left(\partial_{x} \mathbf{u}\right)_{h y b}=f\left(M_{l o c}\right) \mathbf{D}\left(\partial_{x} \mathbf{u}\right)_{u p w}+\left(1-f\left(M_{l o c}\right)\right) \mathbf{D}\left(\partial_{x} \mathbf{u}\right)_{c e n t},
$$

where $\mathbf{D}\left(\partial_{x} \mathbf{u}\right)_{u p w}$ is the upwind spatial discretization $(24)$ and $\mathbf{D}\left(\partial_{x} \mathbf{u}\right)_{\text {cent }}$ is the centered one. $M_{l o c}$ is the local Mach number, which can be computed on the numerical solution at the previous time step. The function $f\left(M_{l o c}\right)$ has to meet the criterion $0 \leq f\left(M_{l o c}\right) \leq 1$. Two different $f$ are adopted:

- minimum function: $f\left(M_{l o c}\right)=\min \left\{1, M_{l o c}\right\}$

- arctangent function: $f\left(M_{l o c}\right)=\frac{\arctan \left(M_{l o c}\right)}{\pi / 2}$.

The two choices for $f$ have proved to be equivalent in the numerical results. The hybrid spatial discretization reads:

$$
\left\{\begin{array}{l}
\mathbf{D}\left(\partial_{x} \mathbf{v}\right)_{h y b}=\frac{1}{2 \Delta x}\left(\mathbf{v}_{i+1}-\mathbf{v}_{i-1}\right)-\frac{f\left(M_{l o c}\right)}{2 \Delta x} \mathbf{A}^{1 / 2}\left(\boldsymbol{\psi}_{i+1}-2 \boldsymbol{\psi}_{i}+\boldsymbol{\psi}_{i-1}\right) \\
\mathbf{D}\left(\partial_{x} \boldsymbol{\psi}\right)_{h y b}=\frac{1}{2 \Delta x}\left(\boldsymbol{\psi}_{i+1}-\boldsymbol{\psi}_{i-1}\right)-\frac{f\left(M_{l o c}\right)}{2 \Delta x} \mathbf{A}^{-1 / 2}\left(\mathbf{v}_{i+1}-2 \mathbf{v}_{i}+\mathbf{v}_{i-1}\right)
\end{array}\right.
$$

We underline the fact that combination (30) essentially produces a centered finite difference scheme. An upwind numerical viscosity is introduced inside the scheme when the Mach number is not close to zero. This is shown by analyzing relation (31). The centered scheme is second order accurate, therefore for low Mach flows the convex combination keeps the same accuracy. In the case of smooth solutions, the slopes can be calculated without the introduction of non-linear limiters. In presence of discontinuities, the nonlinear slope limiters could be computed with a predictor-corrector approach. 
The relaxation all-speed scheme is thus derived. The hybrid spatial discretization (31) is adopted with the implicit time discretization (25), having:

$$
\left\{\begin{array}{c}
\frac{\boldsymbol{\psi}^{n+1}-\boldsymbol{\psi}^{n}}{\Delta t}+\frac{1}{2 \Delta x}\left(\mathbf{v}_{i+1}^{n+1}-\mathbf{v}_{i-1}^{n+1}\right)-\frac{f\left(M_{l o c}\right) \mathbf{A}^{1 / 2}}{2 \Delta x}\left(\boldsymbol{\psi}_{i+1}^{n+1}-2 \boldsymbol{\psi}_{i}^{n+1}+\boldsymbol{\psi}_{i-1}^{n+1}\right)=0 \\
\frac{\mathbf{v}^{n+1}-\mathbf{v}^{n}}{\Delta t}+\frac{\mathbf{A}}{2 \Delta x}\left(\boldsymbol{\psi}_{i+1}^{n+1}-\boldsymbol{\psi}_{i-1}^{n+1}\right)-\frac{f\left(M_{l o c}\right) \mathbf{A}^{1 / 2}}{2 \Delta x}\left(\mathbf{v}_{i+1}^{n+1}-2 \mathbf{v}_{i}^{n+1}+\mathbf{v}_{i-1}^{n+1}\right)= \\
\frac{1}{\eta}\left(\mathbf{F}\left(\boldsymbol{\psi}^{n+1}\right)-\mathbf{v}^{n+1}\right) .
\end{array}\right.
$$

This scheme will be referred to as "implicit relaxation scheme" in what follows.

\subsubsection{Linear system structure}

Linearization (26) introduces a coupling among all the equations. Thus, the following linear system has to be solved:

$$
\left\{\begin{array}{l}
\mathbf{M} \Psi^{n+1}+\mathbf{N V}^{n+1}=\mathbf{r} \\
\mathbf{P} \Psi^{n+1}+\mathbf{Q V}^{n+1}=\mathbf{s}
\end{array}\right.
$$

where $\Psi^{n+1}$ and $\mathbf{V}^{n+1}$ are the vectors containing the grid point values of the five conservative and of the five relaxation variables respectively. The matrices structure comes from the spatial discretization defined in Sec. 4.2.2. With our linearization, the implicit stiff source produces additional terms only on the diagonals of the blocks. A constant term proportional to $1 / \eta$ appears on the diagonal of $\mathbf{Q}$ and the Taylor expansion introduces terms on the diagonals of the sub-blocks of $\mathbf{P}$. Therefore the computational effort in the inversion algorithms is not excessively increased.

We consider the case of a 1D problem on a uniform mesh. At first order, the blocks $\mathbf{M}, \mathbf{N}$ and $\mathbf{Q}$ are tridiagonal. Then the inverse of $\mathbf{M}$ can be computed with a direct solver thanks to its structure. System (33) can be split into two linear systems that are solved sequentially by calculating $\mathbf{V}^{n+1}$ and then $\Psi^{n+1}$ in the following way:

$$
\left\{\begin{array}{l}
\mathbf{V}^{n+1}=\left(\mathbf{Q}-\mathbf{P M}^{-1} \mathbf{N}\right)^{-1}\left(\mathbf{s}-\mathbf{P M}^{-1} \mathbf{r}\right) \\
\Psi^{n+1}=\mathbf{M}^{-1}\left(\mathbf{r}-\mathbf{N V}^{n+1}\right)
\end{array}\right.
$$




\subsection{CFL conditions}

Among the wave speeds $(21)$, let $\mu_{\max }=\sqrt{a_{\max }} \geq \max \left|\lambda_{1}\right|, \lambda_{1}$ being the speed of the longitudinal waves (15) and $\mu_{\text {mat }}=\sqrt{a_{\text {mat }}} \geq \max \left|\lambda_{3}\right|=$ $\max \left|u_{1}\right|$. We define two different CFL conditions that can be enforced to choose the time step.

Definition 4.1. The acoustic Courant number is defined on the speed of the fastest wave as $\nu_{a c}=\mu_{\max } \Delta t / \Delta x$. An acoustic CFL condition is enforced by setting $\nu_{a c} \leq 1$.

Definition 4.2. The material Courant number is defined on the speed of the material wave as $\nu_{\text {mat }}=\mu_{m a t} \Delta t / \Delta x$. A material CFL condition is enforced by setting $\nu_{\text {mat }} \leq 1$.

Remark. For system (9), taking $\mu_{\max } \simeq\left|u_{1}+c+u_{\text {iso }}\right|$ (approximation of the longitudinal speed (15)) we get

$$
\nu_{a c} \simeq \frac{\Delta t}{\Delta x}\left|u_{1}\right|\left(1+\frac{1}{M}+\frac{1}{M_{\chi}}\right) \simeq \nu_{m a t}\left(1+\frac{1}{M}+\frac{1}{M_{\chi}}\right) .
$$

For the Euler equations (namely $\chi=0$ and no deformation), taking $\mu_{\max } \simeq$ $\left|u_{1}+c\right|$ we get

$$
\nu_{a c} \simeq \frac{\Delta t}{\Delta x}\left|u_{1}\right|\left(1+\frac{1}{M}\right) \simeq \nu_{m a t}\left(1+\frac{1}{M}\right) .
$$

For standard explicit schemes as the ones of Sec. 4.1, an acoustic CFL condition is needed for stability. As shown by expressions (34)-(35), this constraint provides an extremely small $\Delta t$ when $M \rightarrow 0$.

The implicit scheme (32), instead, is stable without a CFL constraint. Nevertheless, $\Delta t$ has to be chosen according to the required accuracy. Thus, a material CFL condition is recommended in order to reproduce the propagation of material waves. Since the material CFL does not depend on the speed of the fast waves, these waves are not captured in the low Mach limit.

\subsection{Numerical viscosity}

We study the numerical viscosity by applying the "modified equation" method [10] to schemes at first order on linear transport. As it is well-known in literature, we get the modified equation $\partial_{t} u+\mu \partial_{x} u=D \partial_{x x} u$. Here $\mu$ 
is the speed of the wave (for system (17) is defined in (21)). The diffusion coefficient $D$ for the explicit-upwind scheme is the following:

$$
D_{\text {expl-upw }}=\mu \frac{\Delta x}{2}-\mu^{2} \frac{\Delta t}{2}=(1-\nu) \frac{\mu \Delta x}{2},
$$

where $\nu$ is the Courant number, which needs to be acoustic for this scheme. For the implicit-centered scheme, $D$ can be written as:

$$
D_{\text {impl-cent }}=\mu^{2} \frac{\Delta t}{2}=\frac{\mu \Delta x}{2} \frac{\mu \Delta t}{\Delta x}=\nu \frac{\mu \Delta x}{2} .
$$

The two coefficients shows that for $\nu=\nu_{a c} \lesssim 1$ the implicit-centered scheme has a slightly higher diffusion.

The accuracy in approximating the different waves depends on the chosen $\Delta t$ due to numerical viscosity. With our novel implicit scheme, a material CFL is enforced. For gas flows, the diffusion coefficient (37) on material waves becomes:

$$
D_{m a t}=\frac{\mu_{m a t} \Delta x}{2} \frac{\mu_{m a t} \Delta t}{\Delta x} \simeq \frac{\left|u_{1}\right| \Delta x}{2} \nu_{m a t}
$$

This formulation explains how the scheme is accurate on material waves also when enforcing material CFL constraints on $\Delta t$, namely the accuracy is not reduced by a large time step.

Instead, on acoustic waves, the diffusion coefficient (37) reads as follows:

$$
D_{a c}=\frac{\mu_{\max } \Delta x}{2} \frac{\mu_{\max } \Delta t}{\Delta x} \simeq \frac{\left|u_{1}+c\right| \Delta x}{2} \nu_{a c} \simeq \frac{\left|u_{1}+c\right| \Delta x}{2} \nu_{m a t}\left(1+\frac{1}{M}\right)
$$

where we have used relation (35) for the last equality. In the limit $M \rightarrow 0$, the numerical diffusion of the scheme becomes large on acoustic waves when a material CFL condition is used (large $\Delta t$ ). However, in low Mach flows applications, acoustic waves carry a small amount of energy, thus the low accuracy in their approximation can be acceptable. If accuracy on these waves is needed, acoustic CFL constraints on $\Delta t$ can be applied.

Additional viscosity is also introduced by the relaxation method itself. The first order correction (19) shows the dissipative nature of the relaxation system [28]. In the present work, we control this diffusion by taking $\eta$ equal to $10^{-8}$ and by building $\mathbf{A}$ as explained in Sec. 3.1. 


\section{Numerical results}

In this section, the implicit relaxation scheme (32) is validated at all speeds. We show and discuss several tests by carrying out a thorough comparison with the results of the standard relaxation scheme of Sec. 4.1 (for simplicity of notation, we refer to this scheme as "explicit"). In all computations the relaxation parameter is $\eta=10^{-8}$ and $\mathbf{A}$ is built as explained in Sec. 3.1.

We first present steady test cases on the Euler equations (flow in a nozzle) with different state laws. Then, we study the propagation of material waves in compressible media, with the simulation of the full Eulerian model (9).

\subsection{Laval nozzle flow}

The Laval nozzle is a converging-diverging duct. It is widely used for achieving steady supersonic flows in a variety of systems such as rocket motors and wind tunnels. The sketch of a nozzle is drawn in Fig. 1(a).

The simplest analytic model for compressible flow in a Laval nozzle is the quasi one-dimensional duct flow approximation [44]:

$$
\left\{\begin{array}{l}
\partial_{t}(S \rho)+\partial_{x}(S \rho u)=0 \\
\partial_{t}(S \rho u)+\partial_{x}\left(S\left(\rho u^{2}+p\right)\right)=p \partial_{x} S \\
\partial_{t}(S \rho e)+\partial_{x}(S u(\rho e+p))=0
\end{array}\right.
$$

The quasi one-dimensional assumption consists in taking the cross sectional area as a smooth function of the axial coordinate, $S=S(x)$. Hence, all flow variables are functions of the axial coordinate and not of the other coordinates. After a few manipulations, system (40) can be rearranged in such a way that the Euler system is obtained, with a non linear source:

$$
\left\{\begin{array}{l}
\partial_{t} \rho+\partial_{x}(\rho u)=-\rho u \frac{\partial_{x} S}{S} \\
\partial_{t}(\rho u)+\partial_{x}\left(\rho u^{2}+p\right)=-\rho u^{2} \frac{\partial_{x} S}{S} \\
\partial_{t}(\rho e)+\partial_{x}(u(\rho e+p))=-u(\rho e+p) \frac{\partial_{x} S}{S}
\end{array}\right.
$$

Formulations (40)-(41) are equivalent and both conservative because the cross section $S(x)$ of the nozzle is a smooth function of $x$. System (41) can be rewritten in the general framework (9) with no deformation and with an additional a non-linear source depending on $S$. 
We simulate perfect gas and stiffened gas (water) flows through a Laval nozzle. Steady state is reached evolving system (41) in time until the difference of the solution between two consecutive time steps gets under a certain tolerance (this tolerance is usually taken of order $10^{-9}$ ). All the nozzle results are obtained with 512 grid points unless otherwise stated and on the computational domain $[0,1]$. For all computations, we use the function $S(x)$ plotted in Fig. 1(b).

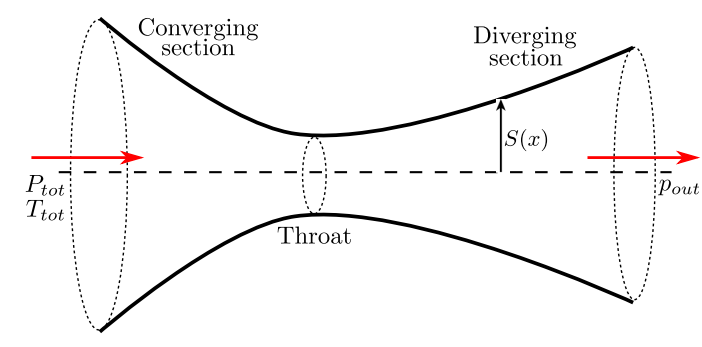

(a) Nozzle

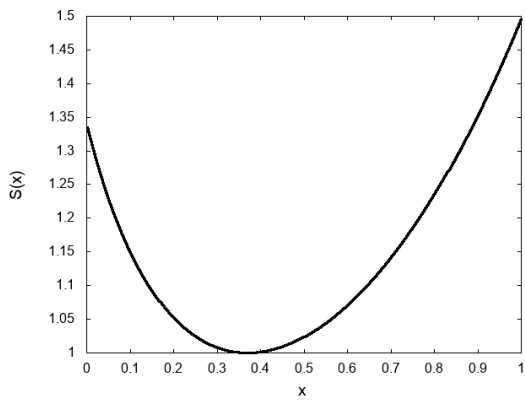

(b) $S(x)$

Figure 1: Left: Laval nozzle general sketch. Right: geometry of the simulated nozzle.

\subsubsection{Perfect gas nozzle flow}

The case of a perfect gas nozzle flow is considered. At the inlet the total pressure (which is given by the Bernoulli principle) and temperature are imposed $P_{\text {tot }}=1 \mathrm{~Pa}$ and $T_{\text {tot }}=1 \mathrm{~K}$. At the outlet, a certain pressure $p_{\text {out }}$ has to be imposed and determines the regime of the nozzle. We study two different configurations:

- test 1: $p_{\text {out }}=0.9 \mathrm{~Pa}$. The flow is subsonic, with a Mach number varying in the range $M \in[0.45 ; 0.7]$;

- test 2: $p_{\text {out }}=0.99999 \mathrm{~Pa}$. The flow is subsonic, with a low Mach number in the range $M \in[4 ; 9] \cdot 10^{-3}$.

We remark that the pressure and temperature values imposed for these configurations are chosen in the spirit of having suitable validation tests of the scheme in different regimes.

In both cases, for the explicit relaxation scheme the results are obtained by enforcing acoustic CFL conditions: $\nu_{a c}=0.9$ at first order and $\nu_{a c}=0.4$ at 


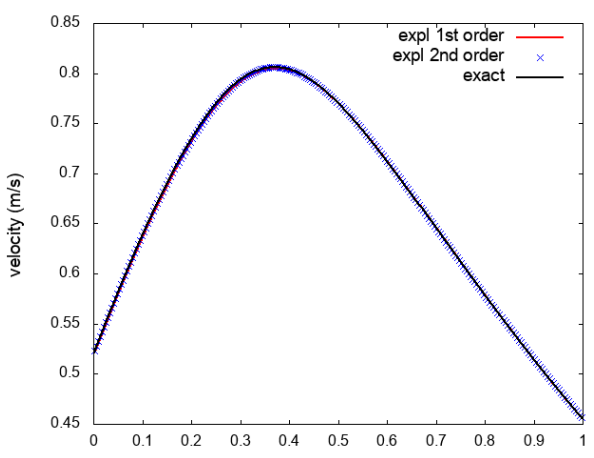

(a) Velocity

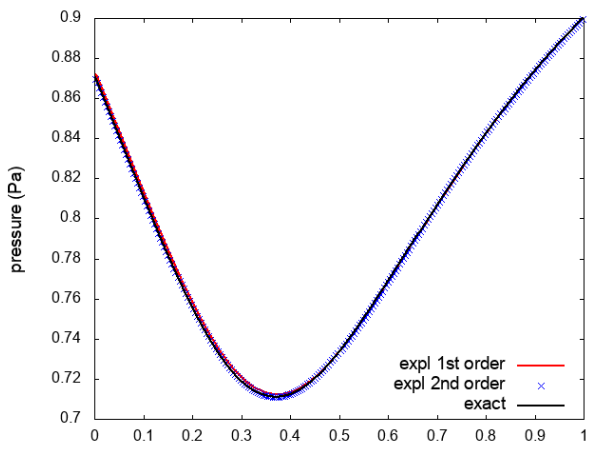

(c) Pressure

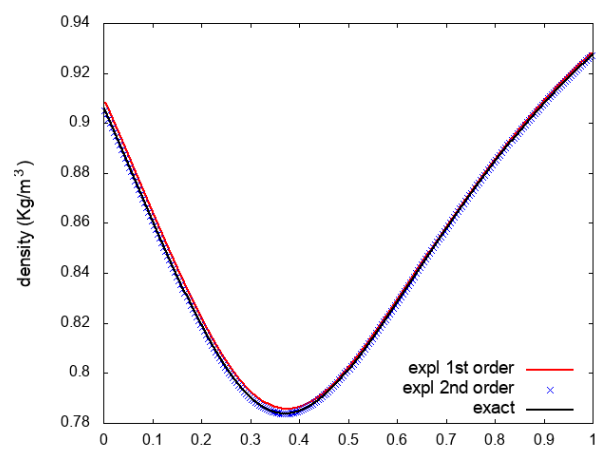

(e) Density

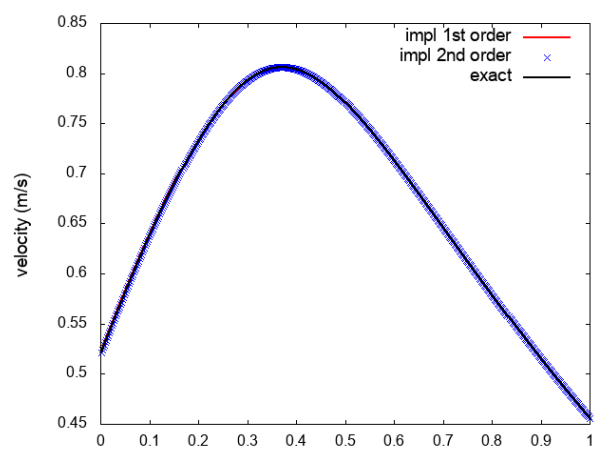

(b) Velocity

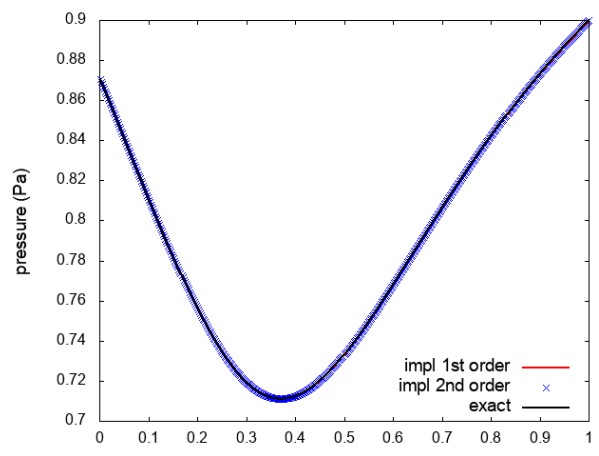

(d) Pressure

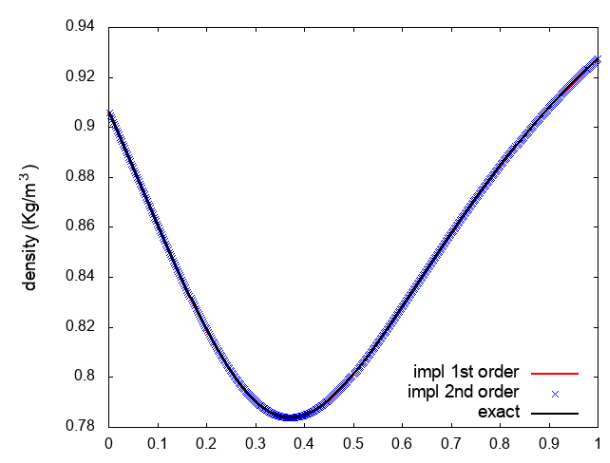

(f) Density

Figure 2: Perfect gas nozzle flow test 1: velocity, pressure and density. Left column: explicit relaxation scheme. Right column: implicit relaxation scheme.

second order. For the implicit scheme, instead, the constraint is not required and the presented results are obtained with $\nu_{a c}=100$. 


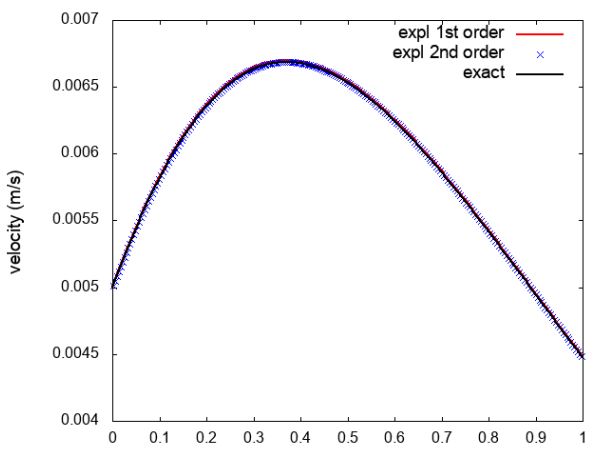

(a) Velocity

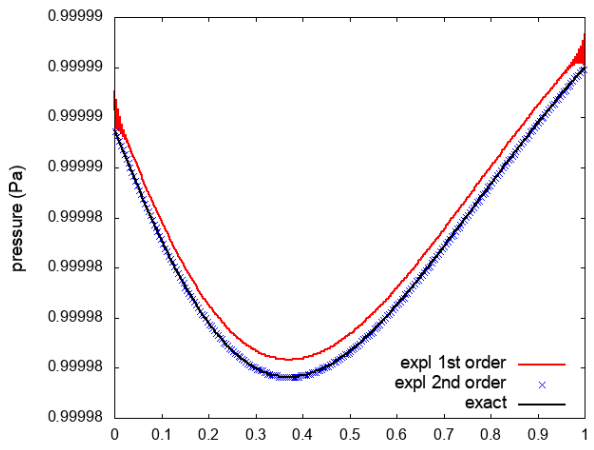

(c) Pressure

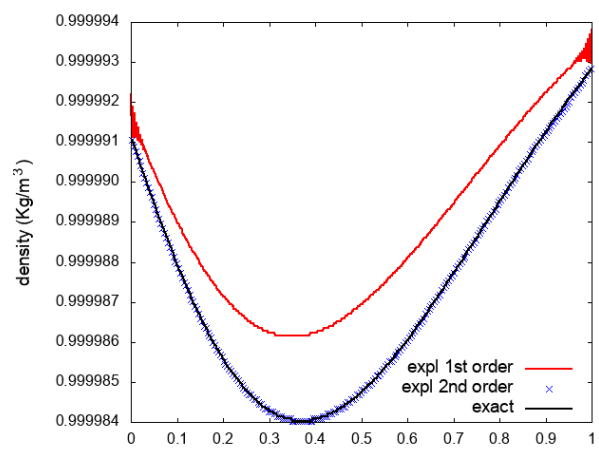

(e) Density

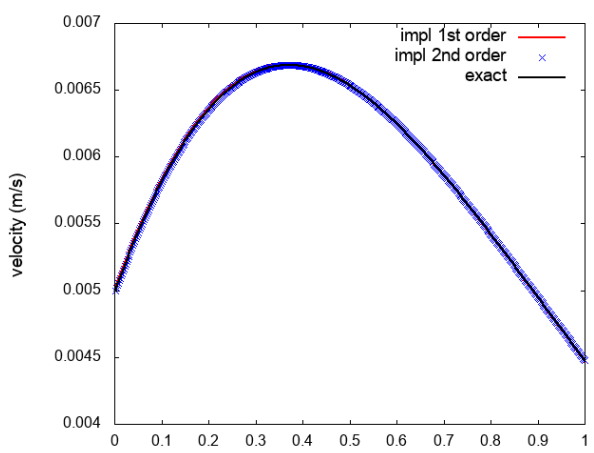

(b) Velocity

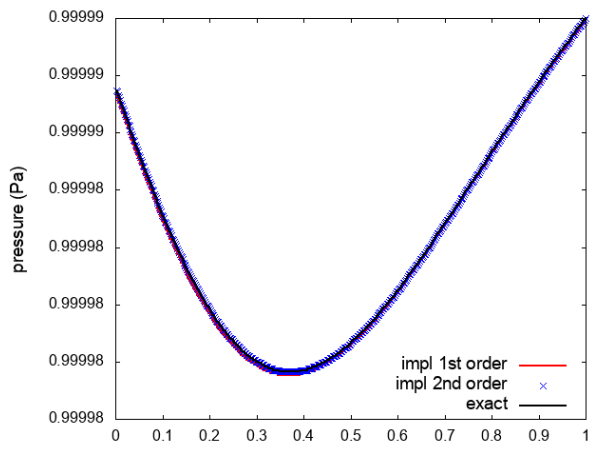

(d) Pressure

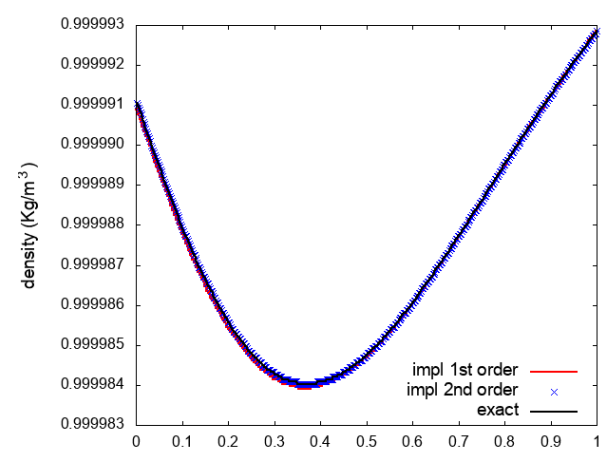

(f) Density

Figure 3: Perfect gas nozzle flow test 2: velocity, pressure and density. Left column: explicit relaxation scheme. Right column: implicit relaxation scheme.

The results of test 1 are presented in Fig. 2. Here we compare the velocity, pressure and density profiles obtained with the standard relaxation 
scheme of Sec. 4.1 (left column) and with the novel implicit scheme (right column). The simulated flow is subsonic, with a Mach number close to one. The two schemes produce very similar results. The convergence analysis for this test (see Fig. 4(a)) shows that the implicit scheme is more precise than the explicit scheme at order one. Nevertheless, at second order, the precision is the same for the two schemes.

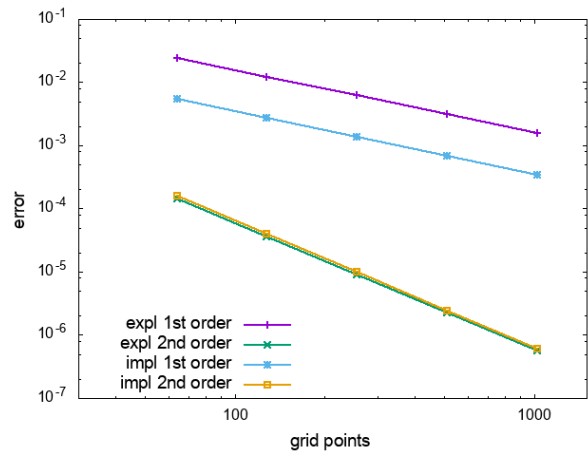

(a) Test 1

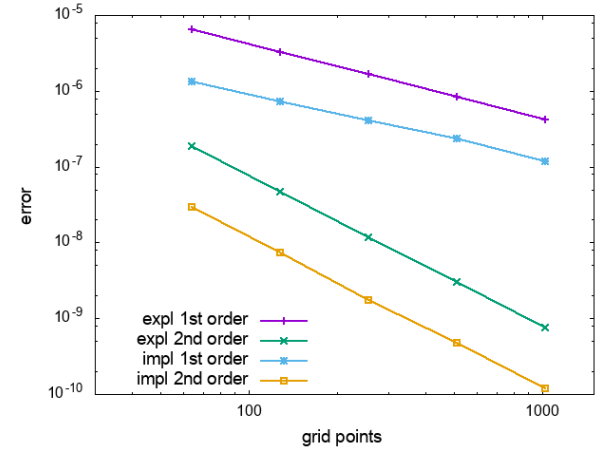

(b) Test 2

Figure 4: Perfect gas nozzle flow: $L_{\infty}$ numerical error on pressure. Left panel: gas nozzle flow test 1. Right panel: gas nozzle flow test 2.

Test 2 is a low Mach flow simulation. The results are presented in Fig. 3, where again the comparison between the results of the two schemes is carried out. The density and pressure profiles obtained with the explicit scheme at first order are shifted with respect to the exact solution and they present some oscillations at the boundaries. This is due to the wrong numerical viscosity of the upwind spatial discretization. Instead, the implicit relaxation scheme is able to overcome these problems: the pressure and the density curves are superimposed to the exact ones.

The convergence analysis of Fig. 4(b) confirms these observations. The implicit scheme is more precise if compared to the explicit scheme, at both first and second order. This is due to the adopted spatial discretization (31), which provides the correct numerical viscosity also when solving low Mach flows. The two proposed tests prove that the novel implicit scheme can provide very accurate solutions in different regimes. The computational effort of the two schemes for the case of 512 grid points is compared in Table 3. The implicit scheme is more expensive mainly because of the simple direct linear solver used. The CPU time could be reduced by employing preconditioned 
iterative methods. On the other hand, techniques such as local time stepping and multigrid (see for example $[45,46]$ ) could accelerate the convergence of the explicit scheme for the specific nozzle application. However, these results show that the numerical error on the low Mach solution is reduced of a factor of 10 with the implicit scheme for a given number of grid points.

(a) Test 1

\begin{tabular}{lll}
\hline & Explicit & Implicit \\
\hline error & $2.75 \cdot 10^{-3}$ & $6.75 \cdot 10^{-4}$ \\
CPU time & 15 & 712 \\
iterations & 22667 & 79
\end{tabular}

(b) Test 2

\begin{tabular}{lll}
\hline & Explicit & Implicit \\
\hline error & $1.64 \cdot 10^{-6}$ & $1.95 \cdot 10^{-7}$ \\
CPU time & 78.3 & 552 \\
iterations & 113914 & 51 \\
\hline
\end{tabular}

Table 3: Comparison of numerical error, CPU time and iterations needed to reach convergence for 512 grid points with the two schemes.

(a) Error $\simeq 7.9 \cdot 10^{-7}$

\begin{tabular}{lll}
\hline & Explicit & Implicit \\
\hline grid points & 2950 & 128 \\
CPU time & 353.1 & 8.1 \\
iterations & 135402 & 48 \\
\hline
\end{tabular}

(b) Error $\simeq 3.8 \cdot 10^{-7}$

\begin{tabular}{lll}
\hline & Explicit & Implicit \\
\hline grid points & 9000 & 256 \\
CPU time & 3464.5 & 64.8 \\
iterations & 448809 & 58 \\
\hline
\end{tabular}

Table 4: Comparison of computational time and iterations needed by the two relaxation schemes to reach the same precision at steady state for test 2 (low Mach flow).

On the other hand, for a given error, the CPU time required by the explicit scheme is much larger than the CPU time of the implicit scheme to reach the same accuracy on the solution. The comparison of computational times, grid points and iterations needed by the two schemes to reach the steady state are shown in Table 4. With the explicit relaxation scheme, the solution appears shifted with respect to the exact solution, due to the incorrect numerical diffusion introduced by upwind differencing (see Fig. 3). Naturally, even in this case the numerical solution is convergent. Thus, the correct numerical solution is recovered only when employing extremely refined grids. Consequently, the computational times increase enormously. We remark that for the computation of the steady state solution, the time dependent problem is solved as an iterative method (such as the Richardson method) until steady state is reached. Therefore, the number of iterations needed to converge corresponds to the number of time steps. 


\subsubsection{Stiffened gas nozzle flow}

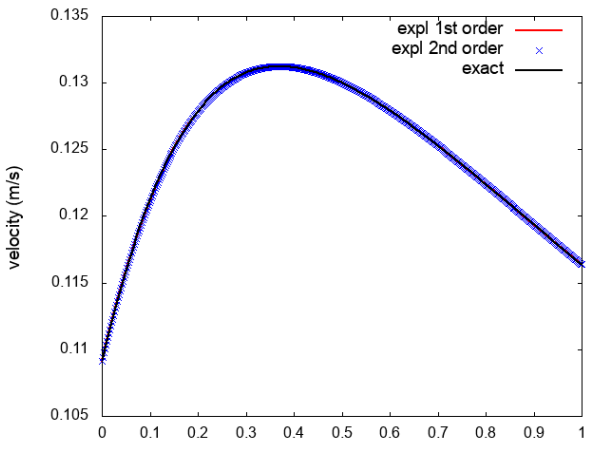

(a) Velocity

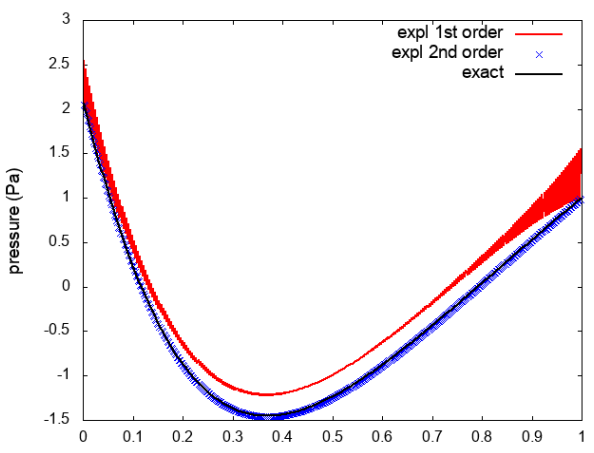

(c) Pressure

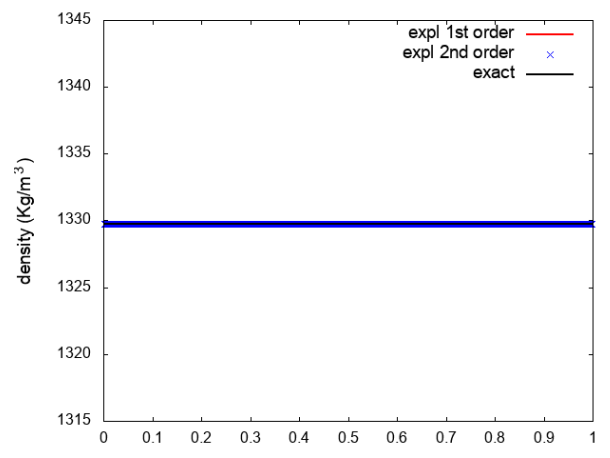

(e) Density

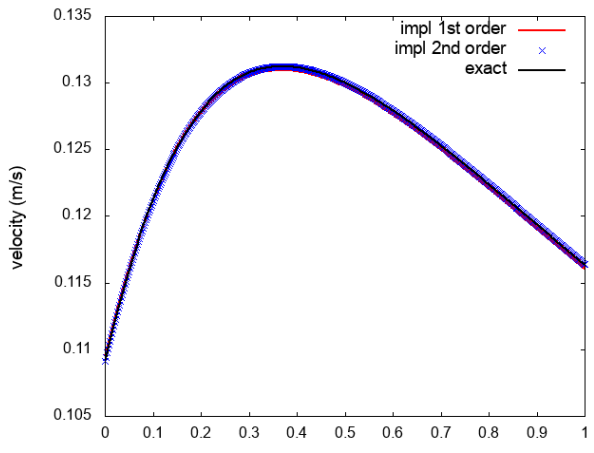

(b) Velocity

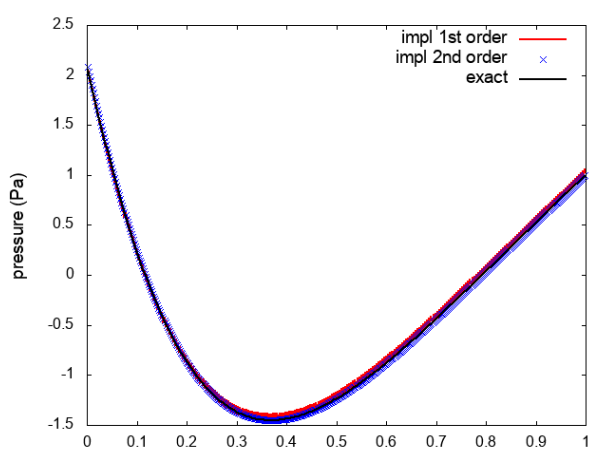

(d) Pressure

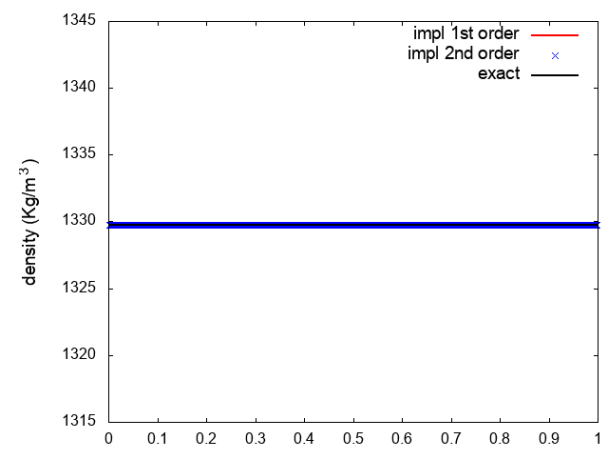

(f) Density

Figure 5: Water nozzle flow: velocity, pressure and density. Left column: explicit relaxation scheme. Right column: implicit relaxation scheme.

With the state law of a stiffened gas, we simulate a water flow inside a 
Laval nozzle. The flow is low Mach and almost incompressible, due to the presence of the $p_{\infty}$ term in the state law. For this simulation, we impose at the inlet $P_{\text {tot }}=10 \mathrm{~Pa}$ and $T_{\text {tot }}=280 \mathrm{~K}$, at the outlet $p_{\text {out }}=1 \mathrm{~Pa}$. The Mach number varies in the interval $M \in[7.26 ; 8.67] \cdot 10^{-5}$ approximately inside the nozzle.

In Fig. 5, the profiles of the density, pressure and velocity obtained with the two schemes are compared with the exact solution. Once again, the oscillations and the shift from the exact solution that can be observed in Fig. $5(\mathrm{c})$ are due to the excessive numerical viscosity of the upwind discretization. The novel implicit relaxation scheme improves the precision of the solution thanks to the hybrid spatial discretization (31).

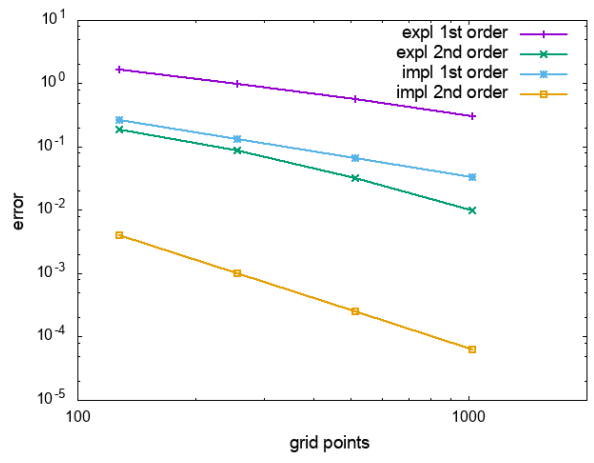

Figure 6: Water nozzle flow: $L_{\infty}$ numerical error on pressure.

The convergence analysis is carried out in Fig. 6. The implicit scheme is always more precise with respect to the explicit one. The convergence rates are the correct ones. In the computations of this test case, the acoustic CFL for the explicit scheme is $\nu_{a c}=0.4$ at first and second order and for the implicit scheme is $\nu_{a c}=100$ at first and second order.

\subsection{Simulation of material waves}

We address the simulation of material waves by solving different Riemann problems in pipes filled with perfect gases, water and hyperelastic solids in different regimes (Table 5). The results are obtained with the explicit relaxation scheme and the novel implicit scheme at first order in all tests.

Initial conditions and parameters are listed in Table 6 , where $L$ is the length of the tube and $x_{0}$ is the initial position of the contact discontinuity. 
The discontinuous initial conditions are smoothed with an arcotangent function in order to overcome the problem of the computation of non-linear flux limiters on the discontinuity.

\begin{tabular}{llllll}
\hline Test & Material & Regime & $\gamma$ & $\begin{array}{l}p_{\infty} \\
(\mathrm{Pa})\end{array}$ & $\begin{array}{l}\chi \\
(\mathrm{Pa})\end{array}$ \\
\hline $\mathbf{1}$ & perfect gas & $M \simeq 0.9$ & 1.4 & 0 & 0 \\
$\mathbf{2}$ & perfect gas & $M \simeq 6 \cdot 10^{-3}$ & 1.4 & 0 & 0 \\
\hline $\mathbf{3}$ & water & $M \simeq 2.5 \cdot 10^{-3}$ & 4.4 & $6.8 \cdot 10^{8}$ & 0 \\
\hline $\mathbf{4}$ & copper & $M \simeq M_{\chi} \simeq \mathcal{O}\left(10^{-3}\right)$ & 4.22 & $3.42 \cdot 10^{10}$ & $5 \cdot 10^{10}$ \\
\hline $\mathbf{5}$ & hyperelastic solid & $M \simeq 3 \cdot 10^{-3}, M_{\chi} \simeq 0.15$ & 4.4 & $6.8 \cdot 10^{8}$ & $8 \cdot 10^{5}$ \\
\hline
\end{tabular}

Table 5: Parameters for the material waves test cases: materials and regime on the contact wave.

\begin{tabular}{llllllllllll}
\hline Test & $\begin{array}{l}L \\
(\mathrm{~m})\end{array}$ & $\begin{array}{l}x_{0} \\
(\mathrm{~m})\end{array}$ & $\begin{array}{l}t_{\text {end }} \\
(\mathrm{s})\end{array}$ & $\begin{array}{l}\rho_{L} \\
\left(\mathrm{Kg} / \mathrm{m}^{3}\right)\end{array}$ & $\begin{array}{l}\rho_{R} \\
\left(\mathrm{Kg} / \mathrm{m}^{3}\right)\end{array}$ & $\begin{array}{l}u_{1, L} \\
(\mathrm{~m} / \mathrm{s})\end{array}$ & $\begin{array}{l}u_{1, R} \\
(\mathrm{~m} / \mathrm{s})\end{array}$ & $\begin{array}{l}u_{2, L} \\
(\mathrm{~m} / \mathrm{s})\end{array}$ & $\begin{array}{l}u_{2, R}, \\
(\mathrm{~m} / \mathrm{s})\end{array}$ & $\begin{array}{l}p_{L} \\
(\mathrm{~Pa})\end{array}$ & $\begin{array}{l}p_{R} \\
(\mathrm{~Pa})\end{array}$ \\
\hline $\mathbf{1}$ & 1 & 0.5 & 0.1644 & 1 & 0.125 & 0 & 0 & 0 & 0 & 1 & 0.1 \\
$\mathbf{2}$ & 1 & 0.5 & 0.25 & 1 & 1 & 0 & 0.008 & 0 & 0 & 0.4 & 0.399 \\
$\mathbf{2 . 1}$ & 400 & 200 & 150 & 1 & 1 & 0 & 0.008 & 0 & 0 & 0.4 & 0.399 \\
\hline $\mathbf{3}$ & 1 & 0.5 & $10^{-4}$ & $10^{3}$ & $10^{3}$ & 0 & 15 & 0 & 0 & $10^{8}$ & $0.98 \cdot 10^{8}$ \\
$\mathbf{3 . 1}$ & 400 & 200 & 0.095 & $10^{3}$ & $10^{3}$ & 0 & 15 & 0 & 0 & $10^{8}$ & $0.98 \cdot 10^{8}$ \\
\hline $\mathbf{4}$ & 2 & 1 & $6 \cdot 10^{-5}$ & $8.9 \cdot 10^{3}$ & $8.9 \cdot 10^{3}$ & 0 & 0 & 0 & 100 & $10^{9}$ & $10^{5}$ \\
$\mathbf{4 . 1}$ & 500 & 250 & 0.04 & $8.9 \cdot 10^{3}$ & $8.9 \cdot 10^{3}$ & 0 & 0 & 0 & 100 & $10^{9}$ & $10^{5}$ \\
\hline $\mathbf{5}$ & 100 & 50 & 0.016 & $1 \cdot 10^{3}$ & $1 \cdot 10^{3}$ & 0 & 10 & 0 & 40 & $10^{8}$ & $0.98 \cdot 10^{8}$ \\
\hline
\end{tabular}

Table 6: Parameters for the material waves test cases: initial state.

For all test cases we use "freeflow" boundary conditions, namely we impose $\left.\frac{\partial \boldsymbol{\psi}}{\partial \mathbf{n}}\right|_{x=0}=\left.\frac{\partial \boldsymbol{\psi}}{\partial \mathbf{n}}\right|_{x=L}=0$ ( $\mathbf{n}$ being the outward normal to the boundary) on the conservative variables. Since $\frac{\partial \mathbf{v}}{\partial \mathbf{n}}=\mathbf{F}^{\prime}(\boldsymbol{\psi}) \frac{\partial \boldsymbol{\psi}}{\partial \mathbf{n}}$, on the relaxation variables we impose $\left.\frac{\partial \mathbf{v}}{\partial \mathbf{n}}\right|_{x=0}=\left.\frac{\partial \mathbf{v}}{\partial \mathbf{n}}\right|_{x=L}=0$.

\subsubsection{Perfect gas}

Test 1 is a Sod shock tube filled with a biatomic perfect gas. The flow is accelerated, characterized by an expansion wave, a contact discontinuity and a shock. The Mach number on the contact wave is $M \simeq 0.9$. The profiles obtained with the explicit and the implicit relaxation schemes are similar, as shown by Fig. 7. Both schemes provide results in good agreement with the exact solution, are oscillation free and have the correct shock strength and speed. The explicit relaxation scheme is solved with $\nu_{a c}=0.4$. For the implicit scheme we impose $\nu_{m a t}=0.3$, corresponding to $\nu_{a c}=0.9$, since the sound speed and the flow velocity are of the same order.

Test 2 is a perfect gas low Mach flow, with $M \simeq 6 \cdot 10^{-3}$ on the contact wave. In the tube, a small pressure ratio and a small velocity on the right are imposed. The gas is expanded in both directions and the contact wave moves very slowly [11]. 


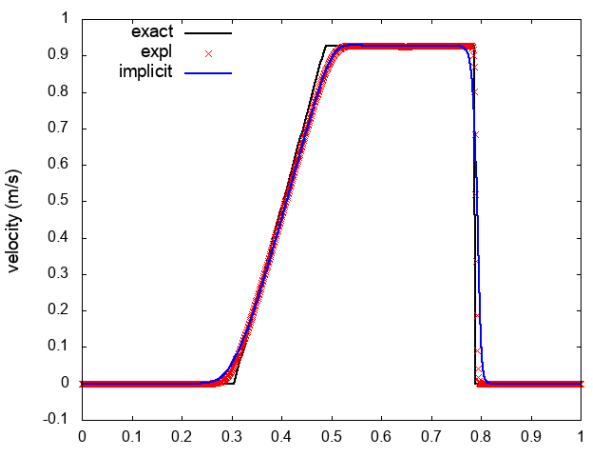

(a) Velocity

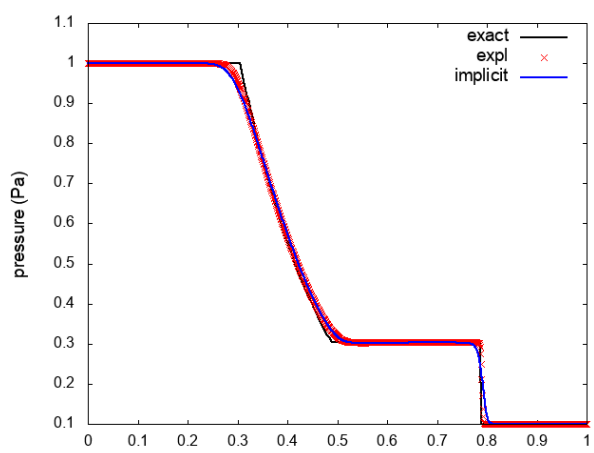

(b) Pressure

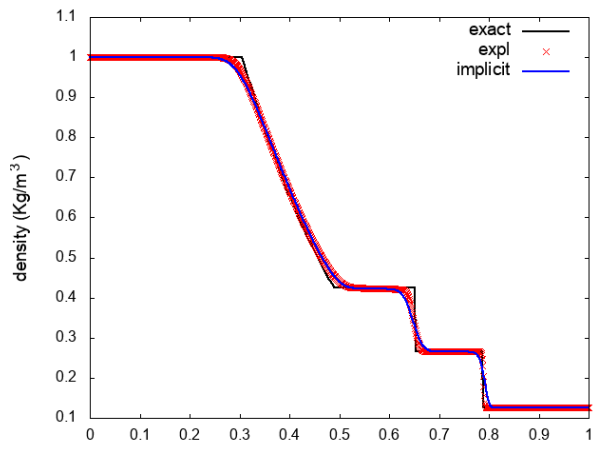

(c) Density

Figure 7: Test 1: Sod shock tube with perfect gas (1000 grid points).

In Fig. 8 we show the influence of the time step on the density profile computed with the implicit scheme. The contact wave is always kept sharp thanks to spatial discretization (31). The numerical viscosity on this wave does not increase when taking a larger time step, as shown by relation (38). All the three waves are accurately reproduced when an acoustic constraint $\nu_{a c}=0.9$ is enforced. With a grid spacing $\Delta x=10^{-3}$, this condition gives a small time step $\Delta t=1.2 \cdot 10^{-3} \mathrm{~s}$ (orange line), thus producing long computational times. Material CFL conditions $\nu_{\text {mat }}=0.1, \nu_{\text {mat }}=0.2$ and $\nu_{\text {mat }}=0.4$ reduce the computational time, since they give $\Delta t=1.1 \cdot 10^{-2}$ (green line), $\Delta t=2.2 \cdot 10^{-2}$ (blue line) and $\Delta t=4.4 \cdot 10^{-2}$ (grey line) respectively. The acoustic waves are smoothed due to the numerical diffusion (39).

The time step of the implicit scheme is not imposed by stability issues, but has to be chosen accordingly to the accuracy needed on the acoustic 


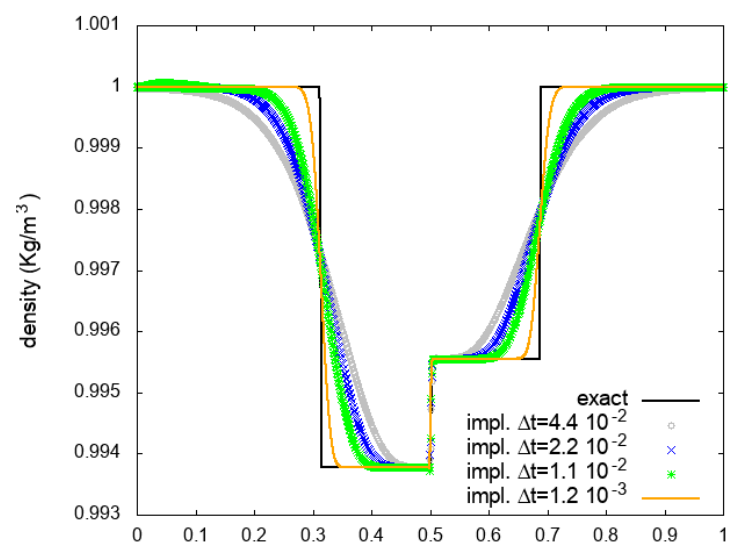

Figure 8: Density profiles for different time steps of the implicit scheme for test 2 (1000 grid points). CFL constraints: $\Delta t=4.4 \cdot 10^{-2}$ given by $\nu_{\text {mat }}=0.4, \Delta t=2.2 \cdot 10^{-2}$ by $\nu_{\text {mat }}=0.2, \Delta t=1.1 \cdot 10^{-2}$ by $\nu_{\text {mat }}=0.1, \Delta t=1.2 \cdot 10^{-3}$ by $\nu_{a c}=0.9$.

waves. If the focus is the approximation of material waves, a material CFL condition can be adopted in order to reduce the computational time. If instead a good resolution of fast waves is needed, the use of an acoustic CFL is recommended.

In Figs. 9(a)-9(b)-9(c) we compare velocity, pressure and density obtained by the implicit scheme with $\nu_{\text {mat }}=0.2$, giving $\Delta t=2.2 \cdot 10^{-2}$ (blue line) and by the explicit scheme, giving $\Delta t=5.3 \cdot 10^{-4}$ (red line). This latter condition gives . After $0.25 \mathrm{~s}$, the contact discontinuity has only moved from $x_{0}=0.5 \mathrm{~m}$ to $x_{0}=0.501 \mathrm{~m}$, namely it has crossed 1 cell for a grid spacing $\Delta x=10^{-3}$. The implicit scheme is consistently more accurate than the explicit scheme in the approximation of the contact wave.

The ability of the scheme in capturing travelling waves is then tested with a simulation for longer times (test 2.1 in Table 6). In Fig. 9(d) we show a zoom on the contact wave in a tube of length $L=400 \mathrm{~m}$. After $150 \mathrm{~s}$ the contact discontinuity has moved from $x_{0}=200 \mathrm{~m}$ to $x_{0}=200.71 \mathrm{~m}$, namely it has crossed 7 cells for the employed grid spacing $\Delta x=10^{-1}$. The explicit relaxation scheme is completely smoothing the contact discontinuity. It is evident that the implicit scheme is superior in capturing the travelling material wave. 


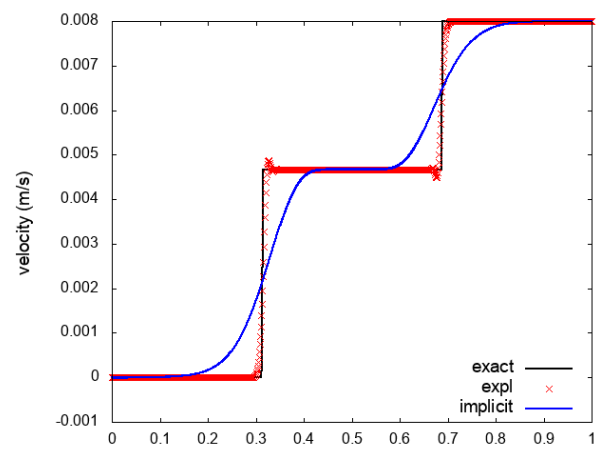

(a) Velocity

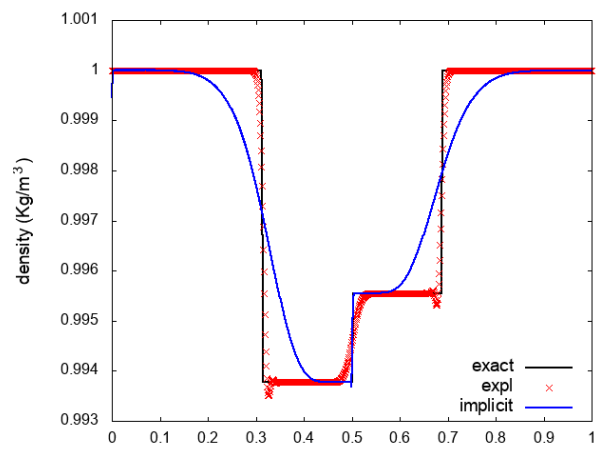

(c) Density

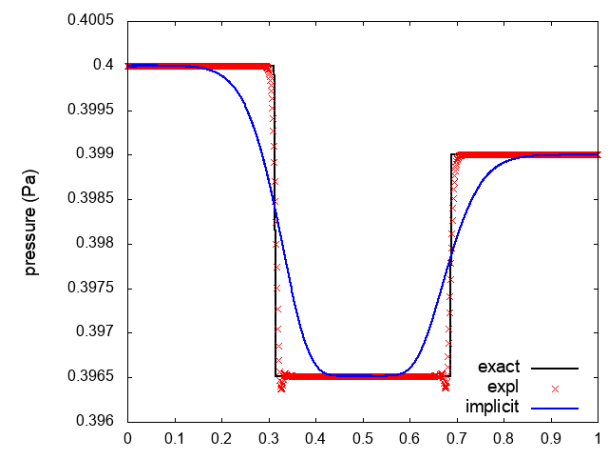

(b) Pressure

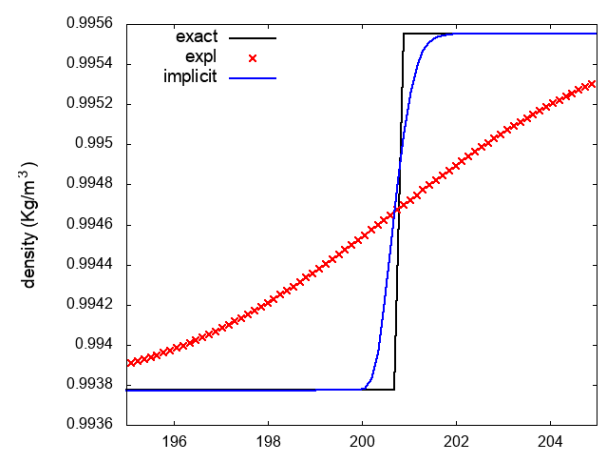

(d) Density after long time

Figure 9: Test 2: Low Mach tube with perfect gas (1000 grid points). Panel (d): zoom on the material wave obtained for test 2.1 (4000 grid points).

\subsubsection{Stiffened gas}

Test 3 simulates a water flow in a pipe where a very small pressure ratio is imposed. The Mach number on the contact wave is $M \simeq 2.5 \cdot 10^{-3}$. In Figs. 10(a)-10(b)-10(c), the explicit scheme presents some small oscillations on the rarefactions due to the stiffness of the problem. These results are obtained with $\nu_{a c}=0.4$ having $\Delta t=2.14 \cdot 10^{-7} \mathrm{~s}$ on a grid of 1000 points. The results of the implicit scheme are obtained with a material CFL $\nu_{\text {mat }}=0.15$, which gives a time step $\Delta t=9.4 \cdot 10^{-6}$ s for the chosen grid. The contact discontinuity is kept sharp and has the correct speed. As expected, the acoustic waves are smoothed due to the large time step. In these results the contact wave has moved from $x_{0}=0.5$ to $x_{0}=0.5008 \mathrm{~m}$, which means that on the chosen grid it has not even crossed one cell yet.

The density profile in Fig. 10(d) is computed after long times (test 3.1: 


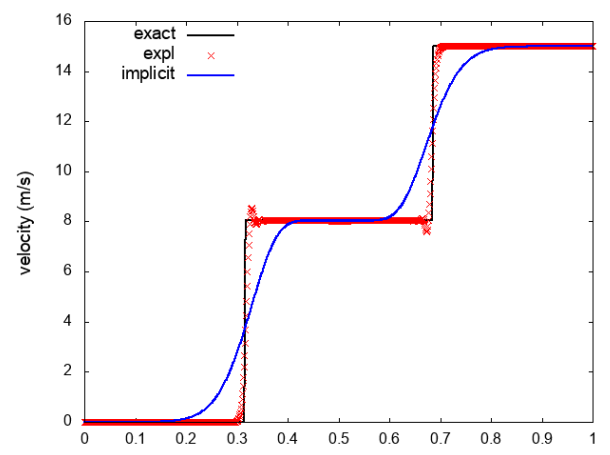

(a) Velocity

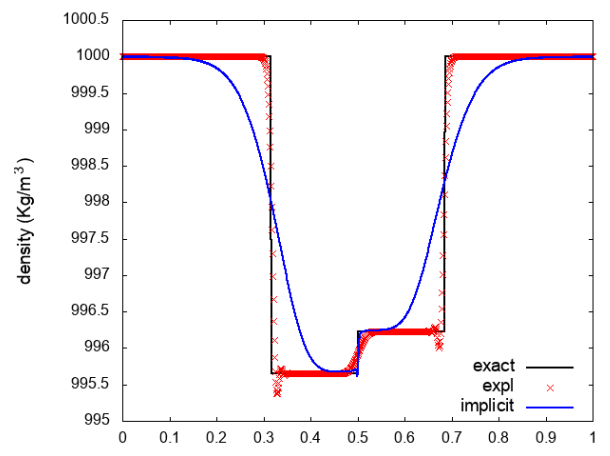

(c) Density

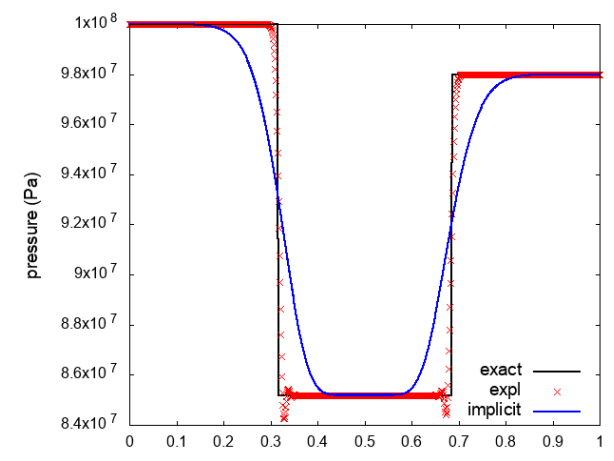

(b) Pressure

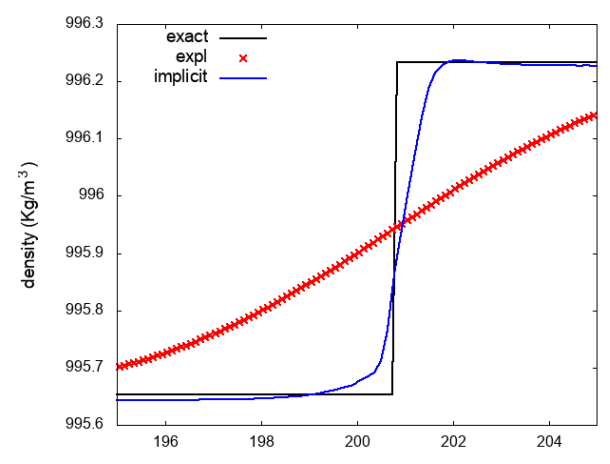

(d) Density after long time

Figure 10: Test 3: tube with water (1000 grid points). Panel (d): zoom on the material wave profile obtained for test 3.1 (4000 grid points).

tube of $L=400 \mathrm{~m})$. At time $t=0.095 \mathrm{~s}$ the contact wave has moved from $x_{0}=200 \mathrm{~m}$ to $x_{0}=200.76 \mathrm{~m}$, namely it has crossed 8 cells for a grid spacing $\Delta x=10^{-1}$. The explicit relaxation scheme is not reproducing the travelling material wave. Instead, the position and the velocity of the wave are captured by the implicit scheme, even if there is not a perfect superimposition due to the stiffness of the problem.

\subsubsection{Hyperelastic solids}

The scheme is tested on the deformation of hyperelastic solids. The 1D system (9) is fully simulated and the isochoric and the volumetric contributions in (5) are both present. Test 4 simulates the deformation of a pipe of length $L=2 \mathrm{~m}$ filled with copper (see Tables 5 and 6 for parameters and initial conditions). Copper is at rest and at higher pressure on the left part. 
A tangential velocity discontinuity is imposed, so five waves appear. On the contact wave, the acoustic Mach number is $M \simeq 2.6 \cdot 10^{-3}$ and the elastic Mach number is $M_{\chi} \simeq 3.15 \cdot 10^{-3}$. Since the two numbers are of the same order, this test is representative of the "shear and acoustic low Mach limit" case, due to intrinsic copper properties $p_{\infty} \simeq \chi \simeq \mathcal{O}\left(10^{10}\right)$.

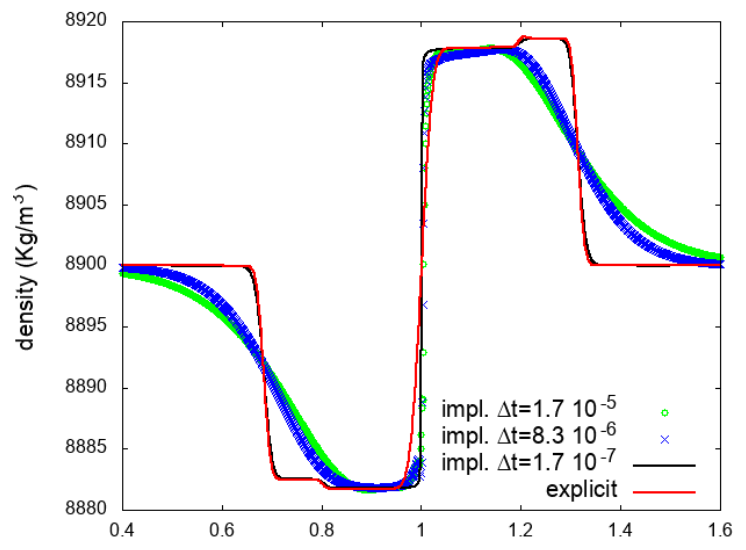

(a) Density

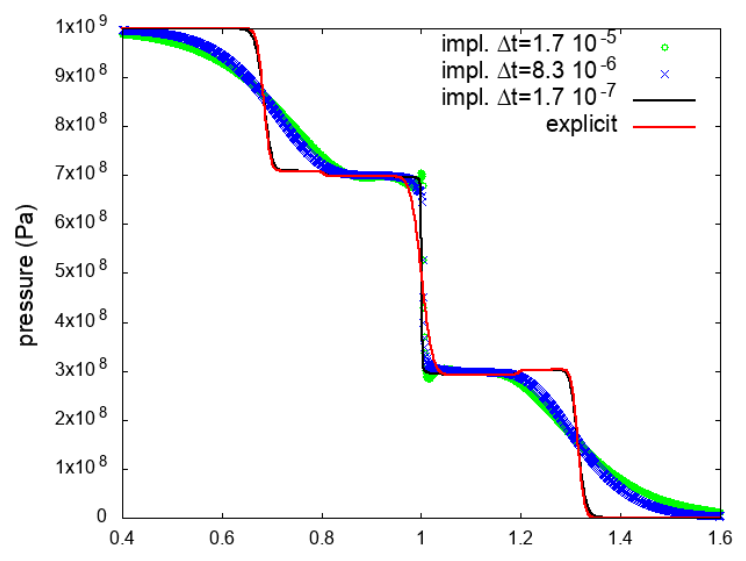

(b) Pressure

Figure 11: Density and pressure profiles for different time steps of the implicit scheme for test 4 (2000 grid points on the domain $[0,2])$. CFL constraints: $\Delta t=1.7 \cdot 10^{-5}$ given by $\nu_{\text {mat }}=0.3, \Delta t=8.3 \cdot 10^{-6}$ by $\nu_{\text {mat }}=0.15, \Delta t=1.7 \cdot 10^{-7}$ by $\nu_{a c}=0.9$.

In Fig. 11 we compare the density and pressure profiles obtained with the implicit scheme for different time steps. All five different waves can be distinguished when the time step is acoustic (black line): the fastest waves 


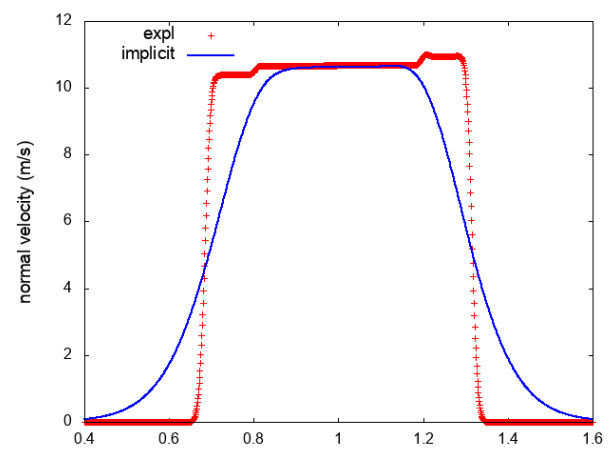

(a) Normal velocity

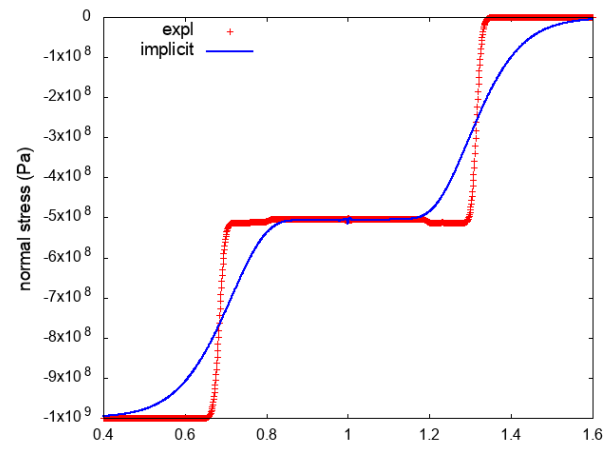

(c) Normal stress

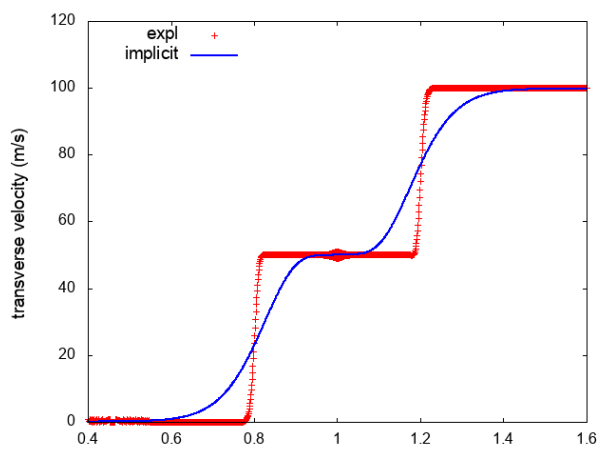

(b) Transverse velocity

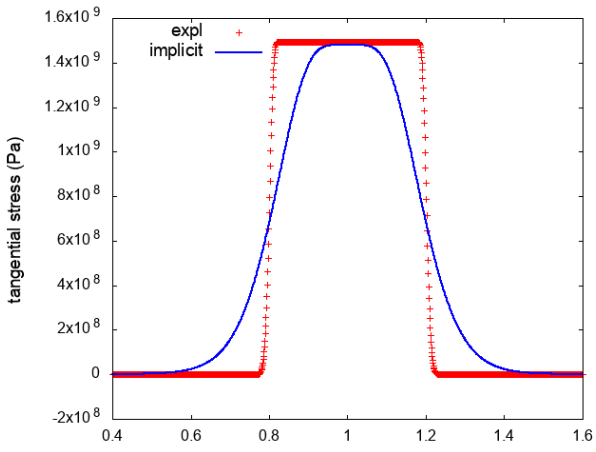

(d) Tangential stress

Figure 12: Test 4: tube with copper (2000 grid points on the domain [0,2]).

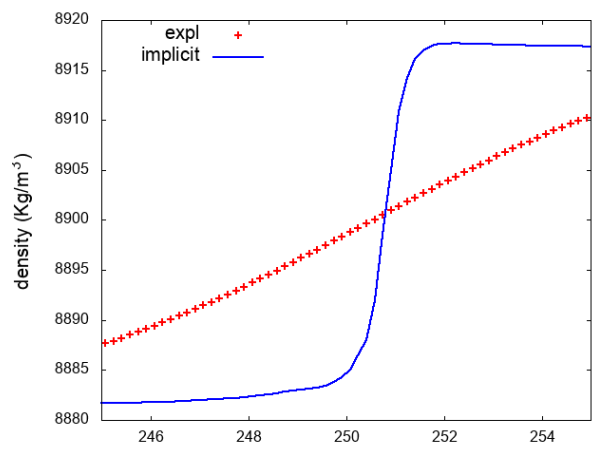

(a) Density

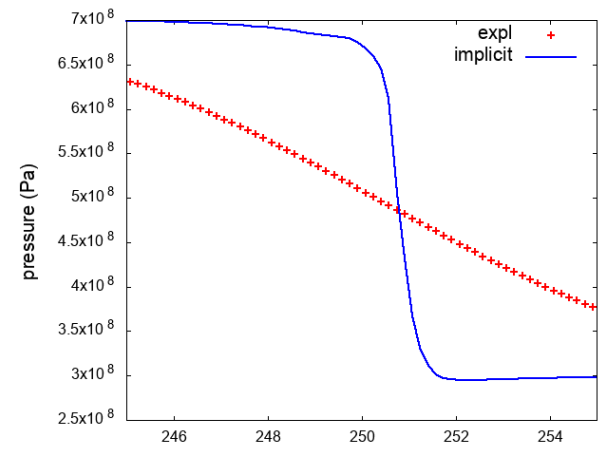

(b) Pressure

Figure 13: Test 4.1: density and pressure for long times (4000 grid points on the domain $[0,500])$. 
are those relative to the normal stress, the middle one is the material wave, and the two intermediate waves are those relative to the tangential (shear) stress. The time step chosen for this simulation is $\Delta t=1.7 \cdot 10^{-7}$, given by enforcing $\nu_{a c}=0.9$. The other results of the implicit scheme are obtained with $\nu_{\text {mat }}=0.15$, giving $\Delta t=8.3 \cdot 10^{-6}$ (blue line) and $\nu_{\text {mat }}=0.3$, giving $\Delta t=1.7 \cdot 10^{-5}$ (green line). With these latter constraints, the number of time steps and, consequently, computational times are reduced. Longitudinal and shear waves are smoothed due to numerical diffusion (39). Nevertheless, for every choice of $\Delta t$, the implicit scheme keeps sharp the material wave, reproducing it more accurately than the explicit scheme. The results of the explicit scheme are calculated with $\nu_{a c}=0.4$ giving $\Delta t=8.7 \cdot 10^{-8}$ (red line). In Fig. 12 we compare the other fields computed by the two schemes. For the implicit scheme, here we use $\nu_{\text {mat }}=0.15$. After $6 \cdot 10^{-5}$ s the contact wave has moved from $x_{0}=1 \mathrm{~m}$ to $x_{0}=1.001 \mathrm{~m}$, not even crossing one cell with the grid spacing $\Delta x=10^{-3}$.

We simulate the same problem for longer times (test 4.1). At time $t=$ $0.04 \mathrm{~s}$, the discontinuity has moved from $x_{0}=250 \mathrm{~m}$ to $x_{0}=250.65 \mathrm{~m}$ in a tube of length $L=500 \mathrm{~m}$. The results in Fig. 13 confirm that the implicit scheme is accurately capturing the travelling material wave, instead the explicit scheme is not reproducing this wave anymore.

As a last peculiar case, we approach the "only acoustic low Mach limit". Test 5 simulates the deformation of an hyperelastic material characterized by $p_{\infty}=\mathcal{O}\left(10^{8}\right) \gg \chi=\mathcal{O}\left(10^{5}\right)$ (for the material parameters and the initial conditions see Tables 5 and 6 ). These parameters were specifically chosen to obtain this particular regime. The two Mach numbers on the contact wave are then $M \simeq 3 \cdot 10^{-3}$ and $M_{\chi} \simeq 0.15$. By imposing a tangential velocity on the right, two slow shear waves arise. The material and the shear waves are almost stationary, whereas the longitudinal waves are extremely fast due to the the stiff $p_{\infty}$ term.

The ability of the implicit scheme in reproducing the slow waves is proven in Fig. 14. Here we show the main profiles in the central region of the domain. By observing the transverse velocity, the tangential stress and the pressure, it is clear that the implicit scheme is superior in capturing the shape and the speed of the shear waves, even if small amplitude oscillations are present. The explicit scheme, instead, does not resolve them. In the density profile obtained by the implicit scheme, the presence of the three slow waves is recognizable, whereas the explicit scheme does not make a distinction (Fig. $14(d))$. 


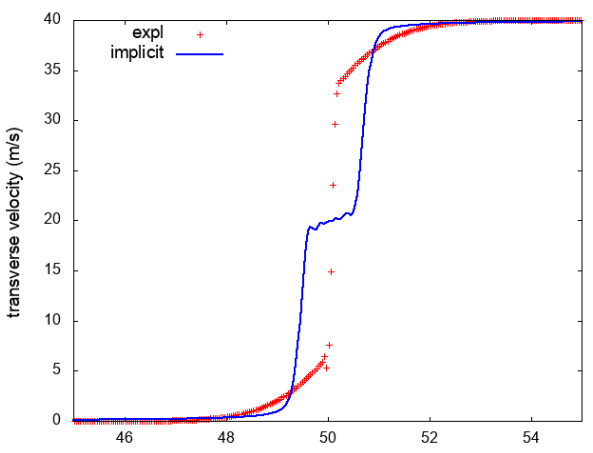

(a) Transverse velocity

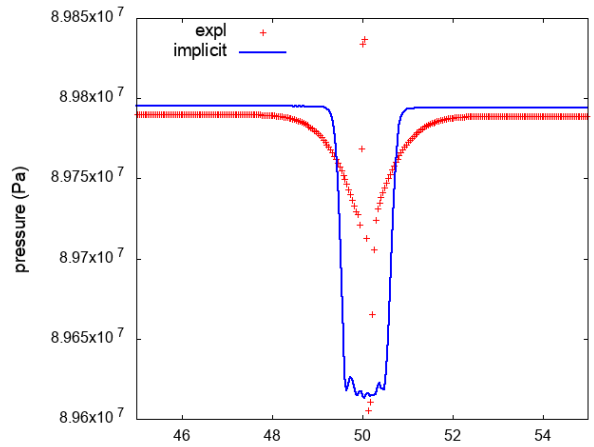

(c) Pressure

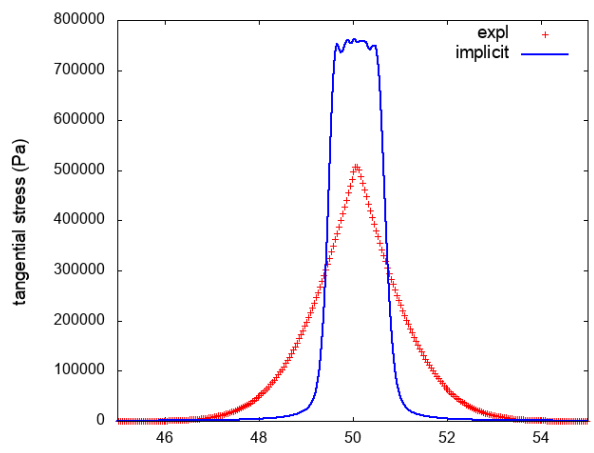

(b) Tangential stress

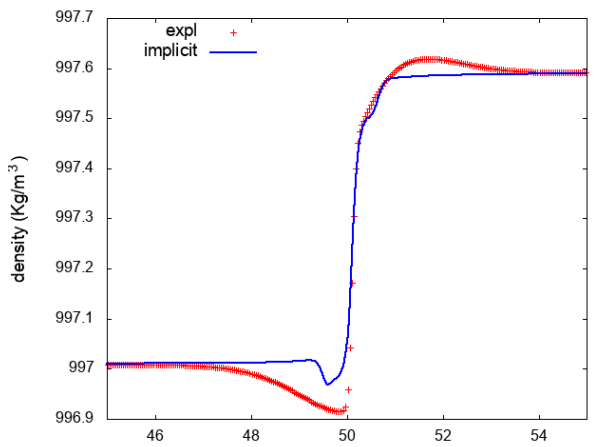

(d) Density

Figure 14: Test 5: transverse velocity, tangential stress, pressure and density (2500 grid points). Zoom on the center of the full domain.

For the explicit scheme, we employ $\nu_{a c}=0.4$, giving $\Delta t=6.89 \cdot 10^{-6} \mathrm{~s}$ on a grid spacing $\Delta x=4 \cdot 10^{-2}$. The results of the implicit scheme are obtained with a time step $\Delta t=2.325 \cdot 10^{-4} \mathrm{~s}$, which is around 300 times bigger than the explicit $\Delta t$. A small time step is here needed due to the presence of three slow waves, which remain very close to each other. In order to limit the oscillations $\Delta t$ could be further reduced.

\section{Conclusions and future developments}

We have proposed an all-speed relaxation scheme for the numerical simulation of compressible materials. The scheme has proved to be accurate in the computation of steady state solutions and also in the approximation of material waves in different Mach regimes. The correct numerical viscosity 
is recovered at all speeds, as shown by the nozzle flow tests. The scheme is applicable to compressible flows inside gases, liquids and hyperelastic solids. We have shown a consistent improvement in the approximation of material waves at slow velocity with respect to explicit schemes.

In the future the scheme will be extended to solve two and three dimensional material wave propagation cases. The all-speed property will allow the simulation of heterogeneous multimaterial problems where the speeds can vary consistently from one medium to another.

\section{Acknowledgments}

G. Puppo and E. Abbate were partially supported by the "National Group for Scientific Computation (GNCS-INDAM), 2016". E. Abbate acknowledges the support of the Università Italo-Francese/Université FrancoItalienne ("Bando Vinci 2016").

\section{References}

[1] S. Godunov, E. Romenskii, Elements of continuum mechanics and conservation laws, Springer Science \& Business Media, 2013.

[2] B. J. Plohr, D. H. Sharp, A conservative Eulerian formulation of the equations for elastic flow, Advances in Applied Mathematics 9 (4) (1988) 481-499.

[3] B. J. Plohr, D. H. Sharp, A conservative formulation for plasticity, Advances in Applied Mathematics 13 (4) (1992) 462-493.

[4] Y. Gorsse, A. Iollo, T. Milcent, H. Telib, A simple Cartesian scheme for compressible multimaterials, Journal of Computational Physics 272 (2014) 772-798.

[5] A. de Brauer, A. Iollo, T. Milcent, A Cartesian scheme for compressible multimaterial models in 3d, Journal of Computational Physics 313 (2016) 121-143.

[6] S. Klainerman, A. Majda, Compressible and incompressible fluids, Communications on Pure and Applied Mathematics 35 (5) (1982) 629-651. 
[7] G. Métivier, S. Schochet, The incompressible limit of the non-isentropic Euler equations, Archive for rational mechanics and analysis 158 (1) (2001) 61-90.

[8] R. Danchin, Low Mach number limit for viscous compressible flows, ESAIM: Mathematical Modelling and Numerical Analysis 39 (3) (2005) 459-475.

[9] F. Miczek, F. K. Röpke, P. V. Edelmann, New numerical solver for flows at various Mach numbers, Astronomy \& Astrophysics 576 (2015) A50.

[10] R. J. LeVeque, Numerical methods for conservation laws, Vol. 132, Springer, 1992.

[11] E. F. Toro, Riemann solvers and numerical methods for fluid dynamics: a practical introduction, Springer Science \& Business Media, 2013.

[12] H. Guillard, C. Viozat, On the behaviour of upwind schemes in the low Mach number limit, Computers \& fluids 28 (1) (1999) 63-86.

[13] S. Dellacherie, Analysis of Godunov type schemes applied to the compressible Euler system at low Mach number, Journal of Computational Physics 229 (4) (2010) 978-1016.

[14] X. Li, C. Gu, An all-speed Roe-type scheme and its asymptotic analysis of low Mach number behaviour, Journal of Computational Physics 227 (10) (2008) 5144-5159.

[15] B. Van Leer, W. Lee, P. Roe, Characteristic time-stepping or local preconditioning of the Euler equations, in: 10th Computational Fluid Dynamics Conference, Vol. 1, 1991, pp. 260-282.

[16] C. Viozat, Implicit upwind schemes for low Mach number compressible flows, Ph.D. thesis, Inria (1997).

[17] W. Barsukow, P. V. Edelmann, C. Klingenberg, F. Miczek, F. K. Roepke, A numerical scheme for the compressible low-Mach number regime of ideal fluid dynamics, arXiv preprint arXiv:1612.03910.

[18] A. J. Chorin, A numerical method for solving incompressible viscous flow problems, Journal of computational physics 2 (1) (1967) 12-26. 
[19] E. Turkel, Preconditioned methods for solving the incompressible and low speed compressible equations, Journal of computational physics 72 (2) (1987) 277-298.

[20] E. Turkel, V. Vatsa, Local preconditioners for steady state and dual time-stepping, Mathematical Modeling and Numerical Analysis, ESAIM: M2AN 39 (2005) 515-536.

[21] R. Klein, Semi-implicit extension of a Godunov-type scheme based on low Mach number asymptotics i: One-dimensional flow, Journal of Computational Physics 121 (2) (1995) 213-237.

[22] P. Degond, M. Tang, All speed scheme for the low Mach number limit of the isentropic Euler equation, arXiv preprint arXiv:0908.1929.

[23] F. Cordier, P. Degond, A. Kumbaro, An asymptotic-preserving all-speed scheme for the Euler and Navier-Stokes equations, Journal of Computational Physics 231 (17) (2012) 5685-5704.

[24] S. Noelle, G. Bispen, K. Arun, M. Lukacova-Medvidova, C. Munz, An asymptotic preserving all Mach number scheme for the Euler equations of gas dynamics, SIAM J. Sci. Comput.

[25] J. Haack, S. Jin, J. Liu, An all-speed asymptotic-preserving method for the isentropic Euler and Navier-Stokes equations, Communications in Computational Physics 12 (04) (2012) 955-980.

[26] C. Berthon, R. Turpault, Asymptotic preserving HLL schemes, Numerical methods for partial differential equations 27 (6) (2011) 1396-1422.

[27] C. Chalons, M. Girardin, S. Kokh, Large time step and asymptotic preserving numerical schemes for the gas dynamics equations with source terms, SIAM Journal on Scientific Computing 35 (6) (2013) A2874A2902.

[28] S. Jin, Z. Xin, The relaxation schemes for systems of conservation laws in arbitrary space dimensions, Communications on Pure and Applied Mathematics 48 (3) (1995) 235-276.

[29] L. Pareschi, G. Russo, Implicit-explicit Runge-Kutta schemes and applications to hyperbolic systems with relaxation, Journal of Scientific computing 25 (1-2) (2005) 129-155. 
[30] A. De Brauer, Simulation de modèles multi-matériaux sur maillage cartésien, Ph.D. thesis, Université de Bordeaux (2015).

[31] G. A. Holzapfel, Nonlinear solid mechanics: a continuum approach for engineering science, Meccanica 37 (4) (2002) 489-490.

[32] T. Liu, Hyperbolic conservation laws with relaxation, Communications in Mathematical Physics 108 (1) (1987) 153-175.

[33] F. Bouchut, Nonlinear stability of finite volume methods for hyperbolic conservation laws: And well-balanced schemes for sources, Springer Science \& Business Media, 2004.

[34] I. Suliciu, On the thermodynamics of rate-type fluids and phase transitions. i. Rate-type fluids, International journal of engineering science 36 (9) (1998) 921-947.

[35] R. LeVeque, M. Pelanti, A class of approximate Riemann solvers and their relation to relaxation schemes, Journal of Computational Physics 172 (2) (2001) 572-591.

[36] C. Chalons, F. Coquel, C. Marmignon, Well-balanced time implicit formulation of relaxation schemes for the Euler equations, SIAM Journal on Scientific Computing 30 (1) (2008) 394-415.

[37] A. Chalabi, Y. Qiu, Relaxation schemes for hyperbolic conservation laws with stiff source terms: Application to reacting Euler equations, Journal of scientific computing 15 (4) (2000) 395-416.

[38] G. B. Whitham, Linear and nonlinear waves, Vol. 42, John Wiley \& Sons, 2011.

[39] S. Boscarino, G. Russo, On a class of uniformly accurate IMEX RungeKutta schemes and applications to hyperbolic systems with relaxation, SIAM Journal on Scientific Computing 31 (3) (2009) 1926-1945.

[40] S. Boscarino, G. Russo, Flux-explicit IMEX Runge-Kutta schemes for hyperbolic to parabolic relaxation problems, SIAM Journal on Numerical Analysis 51 (1) (2013) 163-190. 
[41] F. Cavalli, G. Naldi, G. Puppo, M. Semplice, High-order relaxation schemes for nonlinear degenerate diffusion problems, SIAM Journal on Numerical Analysis 45 (5) (2007) 2098-2119.

[42] S. Dellacherie, J. Jung, P. Omnes, P.-A. Raviart, Construction of modified Godunov-type schemes accurate at any Mach number for the compressible Euler system, Mathematical Models and Methods in Applied Sciences 26 (13) (2016) 2525-2615.

[43] C. Hirsch, Numerical computation of internal and external flows: The fundamentals of computational fluid dynamics, ButterworthHeinemann, 2007.

[44] G. Benison, E. Rubin, A time-dependent analysis for quasi-onedimensional, viscous, heat conducting, compressible Laval nozzle flows, Journal of Engineering Mathematics 5 (1) (1971) 39-49.

[45] R. Swanson, E. Turkel, C.-C. Rossow, Convergence acceleration of Runge-Kutta schemes for solving the navier-stokes equations, Journal of Computational Physics 224 (1) (2007) 365-388.

[46] A. Jameson, D. Caughey, How many steps are required to solve the Euler equations of steady, compressible flow-In search of a fast solution algorithm, in: 15th AIAA Computational Fluid Dynamics Conference, 2001, p. 2673. 